\title{
Test of the Conserved Vector Current Hypothesis by $\beta$-ray Angular Distribution Measurement in the Mass-8 System
}

\author{
T. Sumikama, ${ }^{1,2}$ K. Matsuta, ${ }^{1}$ T. Nagatomo, ${ }^{3}$ M. Ogura, ${ }^{1}$ \\ T. Iwakoshi, ${ }^{1}$ Y. Nakashima,${ }^{1}$ H. Fujiwara, ${ }^{1}$ M. Fukuda, ${ }^{1}$ \\ M. Mihara, ${ }^{1}$ K. Minamisono, ${ }^{4}$ T. Yamaguchi, ${ }^{5}$ and T. Minamisono ${ }^{6}$ \\ ${ }^{1}$ Department of Physics, Osaka University, \\ 1-1 Machikaneyama, Toyonaka, Osaka 560-0043, Japan \\ ${ }^{2}$ Department of Physics, Faculty of Science and Technology, \\ Tokyo University of Science, Noda, Chiba 278-8510, Japan \\ ${ }^{3}$ Department of Chemistry, International Christian \\ University, Mitaka, Tokyo 181-8585, Japan \\ ${ }^{4}$ National Superconducting Cyclotron Laboratory, \\ Michigan State University, East Lansing, Michigan 48824, USA \\ ${ }^{5}$ Department of Physics, Saitama University, Saitama 338-8570, Japan \\ ${ }^{6}$ Fukui University of Technology, 3-6-1 Gakuen, Fukui 910-8505, Japan
}

(Dated: April 22, 2022)

\begin{abstract}
The $\beta$-ray angular correlations for the spin alignments of ${ }^{8} \mathrm{Li}$ and ${ }^{8} \mathrm{~B}$ have been observed in order to test the conserved vector current (CVC) hypothesis. The alignment correlation terms were combined with the known $\beta$ - $\alpha$-angular correlation terms to determine all the matrix elements contributing to the correlation terms. The weak magnetism term, 7.5 \pm 0.2 , deduced from the $\beta$-ray correlation terms was consistent with the CVC prediction 7.3 \pm 0.2 , deduced from the analog- $\gamma$-decay measurement based on the CVC hypothesis. However, there was no consistent CVC prediction for the second-forbidden term associated with the weak vector current. The experimental value for the second-forbidden term was $1.0 \pm 0.3$, while the CVC prediction was $0.1 \pm 0.4$ or $2.1 \pm 0.5$.
\end{abstract}

PACS numbers: 23.20.En, 23.40.Bw 


\section{INTRODUCTION}

In the standard electroweak model, the weak vector current of nucleons and the isovector part of the electromagnetic current form a single isovector electroweak current [1]. The conserved vector current (CVC) hypothesis is analogous to the electromagnetic current conservation law. The weak vector current is conserved despite the influence of strong interactions such as the contribution from the pion cloud around a nucleon. The minimum conditions required for the CVC hypothesis are the universality of the vector coupling constant $g_{\mathrm{V}}$ and the absence of the induced scalar term $g_{\mathrm{S}}$ in the weak nucleon current. The universality of $g_{\mathrm{V}}$ is confirmed at the level of $1.2 \times 10^{-4}$ and $g_{\mathrm{S}}$ is limited to $m_{e} g_{\mathrm{S}} / 2 M_{n} g_{\mathrm{V}}=-(0.0011 \pm 0.0013)$ from 20 superallowed $0^{+} \rightarrow 0^{+} \beta$ decays [2], where $M_{n}$ and $m_{e}$ are the nucleon and electron masses, respectively. In this article, the coupling constant and the induced term in the weak nucleon current, including $g_{\mathrm{V}}$ and $g_{\mathrm{S}}$, are written following Holstein's expression [3].

In addition, the so-called strong CVC hypothesis demands that the weak vector current is paired with the isovector electromagnetic current. For the isospin triplet state, the strong CVC requires that a matrix element from the weak vector current for $\beta$ decay is identical to one from the isovector electromagnetic current for analog- $\gamma$ decay. To test the strong CVC hypothesis, the weak magnetism term $a_{\mathrm{WM}}^{\beta}$ has been compared with the CVC prediction deduced from the isovector M1 component of the analog- $\gamma$ transition strength [4]. The term $a_{\mathrm{WM}}^{\beta}$ was detected using one of the following: a spectral shape factor, a $\beta$-ray angular correlation with a spin orientation, or a correlation with a delayed $\alpha$ or $\gamma$ ray [4-7]. The experimentally determined $a_{\mathrm{WM}}^{\beta}$ may include a possible $G$-parity irregular term, $g_{\mathrm{II}}$, in the weak axial-vector current; therefore, the strong CVC has been tested under the assumption of $G$-parity conservation. Among those studies, Minamisono et al. [6] determined the most accurate $a_{\mathrm{WM}}^{\beta}$ in the mass $A=12$ system. In their paper, the $g_{\mathrm{II}}$ was determined using the CVC prediction, $a_{\mathrm{WM}}^{\mathrm{CVC}}$, for the weak magnetism term. When $G$-parity conservation was assumed, the strong CVC was confirmed as $a_{\mathrm{WM}}^{\beta} / a_{\mathrm{WM}}^{\mathrm{CVC}}=1.04 \pm 0.03$ [8].

Earlier, the strong CVC in the $A=8$ system was tested using the $\beta$ - $\alpha$ angular correlation terms of ${ }^{8} \mathrm{Li}$ and ${ }^{8} \mathrm{~B}[9-11]$. Among the previous measurements, those by Tribble and McKeown [10, 11] were performed for a wide energy range of $\beta$ rays, and the mirror difference $\delta_{\beta \alpha}^{-}$of the $\beta$ - $\alpha$ angular correlation term was determined. $\delta_{\beta \alpha}^{-}$has a contribution of $a_{\mathrm{WM}}^{\beta}$ and a second-forbidden term $a_{\mathrm{WE} 2}^{\beta}$ associated with the weak vector current. While the Tribble's 
data did not reproduce the kinematic shift term for the angular correlation, the McKeown's data reproduced this term properly, and the result was consistent with the CVC prediction value $\delta_{\mathrm{CVC}}^{-}$[7] as $\delta_{\beta \alpha}^{-} / \delta_{\mathrm{CVC}}^{-}=0.93 \pm 0.03 \pm 0.05$, where the first uncertainty was from the $\beta-\alpha$ measurement and the second one was from the CVC prediction.

The $\beta$-ray angular distributions of ${ }^{8} \mathrm{Li}$ and ${ }^{8} \mathrm{~B}$ are given by a combination of several matrix elements; not only $a_{\mathrm{WM}}^{\beta}$ and $a_{\mathrm{WE} 2}^{\beta}$ but also the Gamow-Teller, axial charge, and second forbidden terms from the axial-vector currents. In spite of this complexity, we previously showed [12] that $a_{\mathrm{WM}}^{\beta}$ and $a_{\mathrm{WE} 2}^{\beta}$ could be determined separately by combining the alignment correlation term and the $\beta$ - $\alpha$ angular correlation term. Thus, the strong CVC can be tested for the second-forbidden transition for the first time.

In our previous letter [12], we reported the measurement of the $\beta$-ray angular correlation term from the spin aligned ${ }^{8} \mathrm{Li}$ and ${ }^{8} \mathrm{~B}\left(J^{\pi}=2^{+}\right)$and the limitation of $g_{\mathrm{II}}$ under the assumption of CVC. In the present study, we reanalyzed the data with the assumption of $G$-parity conservation in order to test the strong CVC hypothesis for the weak magnetism and for the second-forbidden transition separately.

\section{BETA-RAY ANGULAR CORRELATION TERMS AND ANALOG GAMMA DECAY}

The two kinds of $\beta$-ray angular correlation term, i.e., the alignment correlation term and the $\beta$ - $\alpha$ angular correlation term, are similar to each other. The alignment correlation term is associated with the spin alignment of parent nucleus. Because $\beta$-delayed $\alpha$ particles are emitted in the direction perpendicular to the angular momentum of the daughter nucleus ${ }^{8} \mathrm{Be}\left(J^{\pi}=2^{+}\right)$, the $\beta$ - $\alpha$ angular correlation term is associated with the spin alignment of the daughter nucleus. As a result, the alignment correlation term and the $\beta$ - $\alpha$ angular correlation term have the same formula except for the signs of several second-forbidden terms. This complementary relationship allows all the matrix elements to be separately determined, as follows.

The $\beta$-ray angular distribution from purely spin-aligned nuclei is given by $W\left(E, \theta_{I \beta}\right) \propto$ $p E\left(E_{0}-E\right)^{2}\left\{B_{0}(E)+\mathcal{A} B_{2}(E) P_{2}\left(\cos \theta_{I \beta}\right)\right\}$, where $p, E, E_{0}$, and $\theta_{I \beta}$ are the $\beta$-ray momentum, energy, end-point energy, and ejection angle with respect to the spin-orientation axis, respectively. $P_{2}$ is the Legendre polynomial. The ${ }^{8} \mathrm{Li}$ and ${ }^{8} \mathrm{~B}$ nuclei decay to the broad first 
excited state of ${ }^{8} \mathrm{Be}$, thus the end-point energy $E_{0}$ is given as $E_{0}=E_{\max }-E_{x}$. $E_{\max }$ is the energy release during the $\beta$ decay to the ${ }^{8}$ Be ground state, while $E_{x}$ is the excitation energy of ${ }^{8} \mathrm{Be}$. The nuclear-spin alignment $\mathcal{A}=\left(2 a_{+2}-a_{+1}-2 a_{0}-a_{-1}+2 a_{-2}\right) / 2$ is given by the population $a_{m}$ of the magnetic substate $m$, with $\sum a_{m}=1$. The alignment correlation terms $B_{2}(E) / B_{0}(E)$ for ${ }^{8} \mathrm{Li}$ and ${ }^{8} \mathrm{~B}$ are given by $K(E, 0)$ in [3] as

$$
\begin{aligned}
K(E, s)= & -\frac{E}{3 M_{n}}\left[\frac{1}{A} \pm \frac{b}{A c}-\frac{d_{\mathrm{I}}}{A c} \mp \frac{g_{\mathrm{II}}}{g_{\mathrm{A}}}\right. \\
& +\frac{(-)^{s}}{\sqrt{14}}\left\{ \pm \frac{f}{A c} \frac{E_{0}+2 E}{E_{0}}+\frac{3}{2} \frac{j_{2}}{A^{2} c} \frac{E_{0}-2 E}{M_{n}}\right\} \\
& \left.-\frac{3}{\sqrt{35}} \frac{j_{3}}{A^{2} c} \frac{E}{M_{n}}\right],
\end{aligned}
$$

where $g_{\mathrm{A}}$ is the axial-vector coupling constant, $c$ is the Gamow-Teller matrix element, $b$ is the weak magnetism matrix element, $d_{\mathrm{I}}$ is the axial charge, $f$ is the second-forbidden term from the vector current, $j_{2}$ and $j_{3}$ are the second-forbidden terms from the axial-vector current, and $A$ is the mass number of the nucleus. $a_{\mathrm{WM}}$ and $a_{\mathrm{WE} 2}$ are given by the ratios $a_{\mathrm{WM}}=b / A c$ and $a_{\mathrm{WE} 2}=f / A c$. The $\beta-\alpha$ angular correlation term, on the other hand, is given by $W\left(E, \theta_{\beta \alpha}\right) \propto p E\left(E_{0}-E\right)^{2}\left\{1+a_{\mp}(E) \cos \theta_{\beta \alpha}+p_{\mp}(E) \cos ^{2} \theta_{\beta \alpha}\right\}$, where $\theta_{\beta \alpha}$ is the angle between the momenta of $\beta$ and $\alpha$ rays. $a_{\mp}(E)$ is the kinematic shift term associated with the recoil of the daughter nucleus. The $\beta-\alpha$ angular correlation term $p_{\mp}(E)$ is given as $-\frac{2}{3} p_{\mp}(E)=K(E, 1)$, which is also defined by Eq. (1). The difference in the correlation terms between the mirror pair, $\delta_{\text {align. }}^{-}=\left(B_{2} / B_{0}\right)_{{ } \mathrm{Li}}-\left(B_{2} / B_{0}\right)_{{ }_{8} \mathrm{~B}}$ and $\delta_{\beta \alpha}^{-}=(-2 / 3)\left(p_{-}-p_{+}\right)$, consists of only three terms, $b / A c, g_{\mathrm{II}} / g_{\mathrm{A}}$, and $f / A c$. The $b / A c$ term is determined under the assumption that $g_{\mathrm{II}}=0 ; f / A c$ is completely separated from the others as follows:

$$
\begin{aligned}
& \frac{\delta_{\text {align. }}^{-}+\delta_{\beta \alpha}^{-}}{2}=-\frac{2 E}{3 M_{n}} \frac{b}{A c} \\
& \frac{\delta_{\text {align. }}^{-}-\delta_{\beta \alpha}^{-}}{2}=-\frac{2 E}{3 M_{n}} \frac{f}{A c} \frac{E_{0}+2 E}{\sqrt{14} E_{0}} .
\end{aligned}
$$

The $c, b$, and $f$ terms described by reduced matrix elements as follows: $c=g_{\mathrm{A}}\left\langle f\left\|\tau^{ \pm} \boldsymbol{\sigma}\right\| i\right\rangle$, $b=A\left(g_{\mathrm{M}}\left\langle f\left\|\tau^{ \pm} \boldsymbol{\sigma}\right\| i\right\rangle+g_{\mathrm{V}}\left\langle f\left\|\tau^{ \pm} \boldsymbol{L}\right\| i\right\rangle\right)$, and $f=2(2 \pi / 15)^{1 / 2} A M_{n} E_{0} g_{\mathrm{V}}\left\langle f\left\|\tau^{ \pm} r^{2} Y_{2}(\hat{r})\right\| i\right\rangle$, where $g_{\mathrm{M}}$ is the weak magnetism coupling constant in the weak vector current. $c$ is determined from the $\beta$-decay half-lives of ${ }^{8} \mathrm{Li}$ and ${ }^{8} \mathrm{~B}$.

The matrix elements depend on the final state energy which is broadly distributed. The $E_{x}$ dependence of $c$ and $b$ is taken into account by using $R$-matrix theory as described 
in Secs. $\mathrm{VB}$ and $\mathrm{VD}$. The $E_{x}$ dependence of the others is considered as a systematic uncertainty as described in Sec. VD.

The requirement by strong $\mathrm{CVC}$ is that $b$ and $f$ contribute also to the electromagnetic transition from the isobaric analog state in ${ }^{8} \mathrm{Be} . b$ and $f$ are related to the isovector components of the M1 and E2 transition strengths, $\Gamma_{\mathrm{M} 1}^{T=1}$ and $\Gamma_{\mathrm{E} 2}^{T=1}$, i.e. $b=A M_{n}\left\{6 \Gamma_{\mathrm{M} 1}^{T=1} /\left(\alpha E_{\gamma}^{3}\right)\right\}^{1 / 2}$ and $f / b=\sqrt{10 / 3} \delta_{1}[7]$. Here $E_{\gamma}$ is the $\gamma$-ray energy, the fine structure constant $\alpha=1 / 137$, and the $\mathrm{M} 1 / \mathrm{E} 2$ ratio $\delta_{1}=\left(\Gamma_{\mathrm{E} 2}^{T=1} / \Gamma_{\mathrm{M} 1}^{T=1}\right)^{1 / 2}$.

The initial state of the analog- $\gamma$ decay splits into two isospin mixing states with $T=0$ and 1. In addition, the electromagnetic transitions from these states include the isoscalar and isovector components. Two strengths $\Gamma_{\mathrm{M} 1}^{T=1}$ and $\Gamma_{\mathrm{E} 2}^{T=1}$ are the isovector component from the state with $T=1$. The measurement of the $\gamma$ decay from these states and the extraction of $\Gamma_{\mathrm{M} 1}^{T=1}$ and $\Gamma_{\mathrm{E} 2}^{T=1}$ were performed in the previous work by De Braeckeleer et al. [7].

\section{EXPERIMENTAL}

In this section, the experimental details for the alignment correlation term measurement is described. Figure 1 shows the experimental setup, which is essentially similar to the previous experiment for the alignment correlation terms of ${ }^{12} \mathrm{~B}$ and ${ }^{12} \mathrm{~N}[6]$.

\section{A. Production of unstable ${ }^{8} \mathrm{Li}$ and ${ }^{8} \mathrm{~B}$}

The ${ }^{8} \mathrm{Li}\left[{ }^{8} \mathrm{~B}\right]$ nuclei were produced through the nuclear reactions ${ }^{7} \mathrm{Li}(d, p){ }^{8} \mathrm{Li}\left[{ }^{6} \mathrm{Li}\left({ }^{3} \mathrm{He}, n\right)^{8} \mathrm{~B}\right]$. Hereafter, information with the parentheses represent the conditions for ${ }^{8} \mathrm{~B} . \mathrm{A} \mathrm{Li}_{2} \mathrm{O}$ [enriched metal ${ }^{6} \mathrm{Li}$ ] target was bombarded by a deuteron $\left[{ }^{3} \mathrm{He}\right]$ beam at $3.5 \mathrm{MeV}[4.7 \mathrm{MeV}]$ with a typical intensity of $9 \mu \mathrm{A}[40 \mu \mathrm{A}]$. A rotating target, which occupied one third of the circumference of the target rotor, was cooled from inside the holder by a compressed air jet in order to withstand the high-intensity ${ }^{3} \mathrm{He}$ beam, which operated at $4.7 \mathrm{MeV}$ up to 40 $\mu \mathrm{A}$. The pulsed beam was synchronized to the rotational period of $2.4 \mathrm{~s}$. The beam-on and beam-off times were $0.8 \mathrm{~s}$ and $1.6 \mathrm{~s}$, respectively. The target material was vacuum evaporated on a backing ribbon made of molybdenum [phosphor bronze]. Phosphor bronze was used to reduce a Rutherford scattering of ${ }^{3} \mathrm{He}$, which could otherwise have bombarded the recoil catcher and have been an origin of disturbing background activities. A new ion-source 

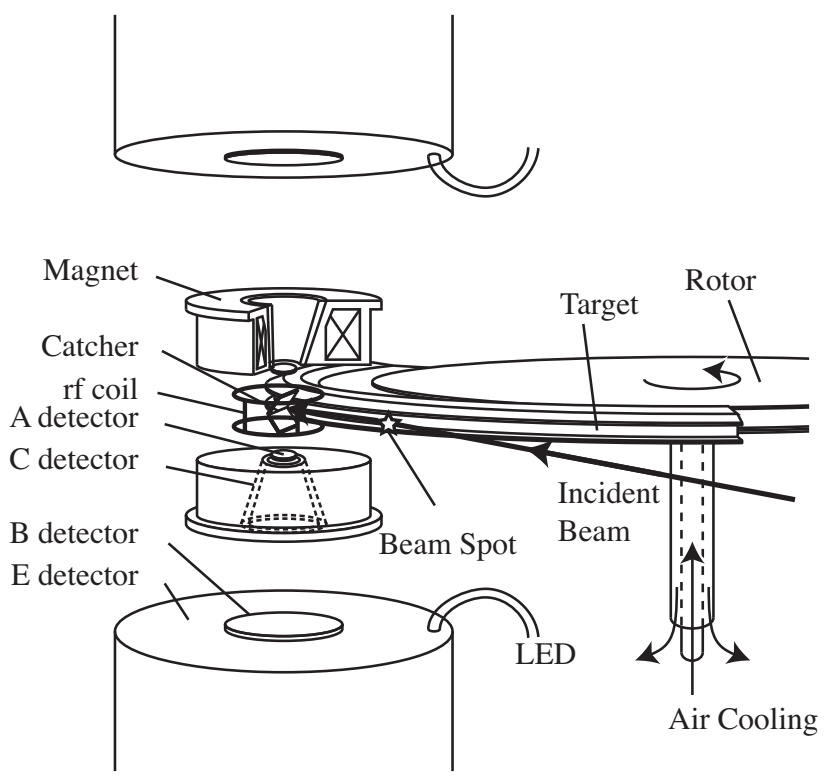

FIG. 1. Schematic view of the experimental setup. The rotational target with an air cooling system was used to reduce the background from the target. The catcher and the rf coil were placed at the center of two telescopes. Each plastic scintillation detector telescope consisted of two thin $\Delta \mathrm{E}$ detectors (A and $\mathrm{B})$, one veto detector $(\overline{\mathrm{C}})$, and one energy detector $(\mathrm{E})$.

bottle, made of glass, was used for the ${ }^{3} \mathrm{He}$ beam to prevent a very weak $\mathrm{HD}^{+}$molecular ion beam from mixing with the ${ }^{3} \mathrm{He}$ beam. The $\mathrm{HD}^{+}$ion beam was formed by $\mathrm{H}_{2}$ and $\mathrm{D}_{2}$ gases oozing out from the inner wall of the ion-source bottle, which were in turn used for the production of $p^{+}$or $d^{+}$beams.

\section{B. Recoil implantation of polarized nuclei}

The recoil angle of the nuclear-reaction products was selected in the range $14^{\circ}-40^{\circ}\left[7^{\circ}\right.$ $\left.18^{\circ}\right]$ to optimize the obtained polarization. The polarized ${ }^{8} \mathrm{Li}\left[{ }^{8} \mathrm{~B}\right]$ nuclei were implanted in $\mathrm{Zn}\left[\mathrm{TiO}_{2}\right.$ (rutile structure)] single crystals by using a recoil energy of $1.7 \mathrm{MeV}[2.3$ $\mathrm{MeV}]$ obtained by the nuclear reaction. The crystals were placed in a static magnetic field $B_{0}$ to maintain the polarization and to manipulate the spin orientation using the $\beta$-NMR technique. The c axis of the single crystals was set parallel to $B_{0}$, which was $60 \mathrm{mT}[230$ $\mathrm{mT}]$. An asymmetry of $\beta$-rays emitted from polarized nuclei was detected by two sets of the counter telescope placed in the opposite direction. The obtained polarization was determined to be $7.2 \%$ [5.4\%] from the $\beta$-ray asymmetry by using the $\beta$-NMR technique. 


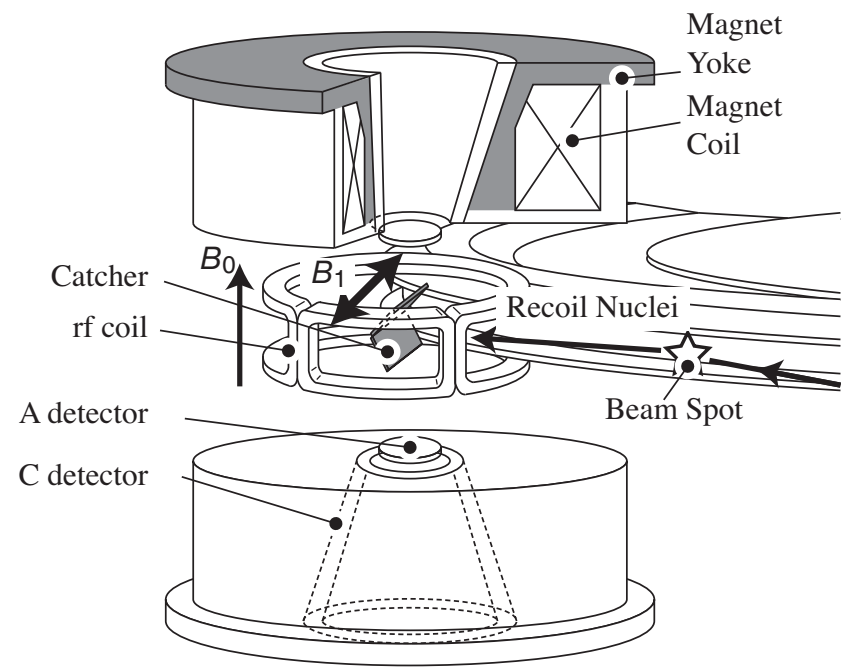

FIG. 2. Focused view of NMR equipment. An rf oscillating magnetic field $B_{1}$, which was applied by the rf coil, is perpendicular to the external magnetic field $B_{0}$.

The recoil catcher consisted of a pair of crystals, which were tilted $45^{\circ}$ with respect to the magnetic field but in opposite directions in order to form a dog-leg shape (similar to a half-opened book) as seen from the side, as shown in Fig. 2, The implantation depth was uniformly distributed at $2.4 \mu \mathrm{m}[3.1 \mu \mathrm{m}]$ from the surface. The recoil nuclei were implanted from the inner side of the two crystals, making the path length and the energy loss of the $\beta$ rays in the catcher less sensitive to the $\beta$-emitter position. The thickness of crystals was $360 \pm 20 \mu \mathrm{m}$ for the guph Zn crystal and $250 \pm 20 \mu \mathrm{m}$ for the gdownh crystal, and $100 \pm 10$ $\mu \mathrm{m}$ for both $\mathrm{TiO}_{2}$ crystals. The systematic uncertainty due to the ambiguity in the thickness was considered as discussed in Sec. IVD.

\section{Spin manipulation}

In order to convert the initial polarization into positive and negative alignments with, ideally, zero polarization, the nuclear spin was manipulated using the NMR technique. The Larmor frequency for spin $J=2$ nucleus splits into four resonance frequencies because of hyperfine interaction between the electric quadrupole moment $Q$ of the implanted nucleus and the electric field gradient (EFG) at an implantation site in the crystal. EFG is defined by $V_{i i}=d^{2} V / d i^{2}$, where $i$ is the principal axes of EFG, i.e., $X, Y$, and $Z, V_{X X}+V_{Y Y}+V_{Z Z}=0$, and $\left|V_{X X}\right| \leq\left|V_{Y Y}\right| \leq\left|V_{Z Z}\right|$. Therefore, once principal axes are chosen, EFG is given by 
two parameters $q=V_{Z Z}$ and $\eta=\left(V_{X X}-V_{Y Y}\right) / V_{Z Z}$. The resonance frequency between two neighboring magnetic substates, $(m-1) \leftrightarrow m$, is given in [13] as

$$
\nu_{m-1 \leftrightarrow m}=\nu_{L}-\frac{\nu_{Q}}{4}\left(3 \cos ^{2} \theta-1+\eta \sin ^{2} \theta \cos 2 \phi\right)(2 m-1)
$$

where $\nu_{L}$ is the Larmor frequency, $\nu_{Q}=e q Q / 4 h$, and $\theta$ and $\phi$ are the Euler angle between the principal axes of EFG and the external magnetic field, respectively.

Populations of two neighboring magnetic substates can be manipulated independently by applying an rf oscillating magnetic field at each frequency. EFG at implantation sites in crystals has been studied by the $\beta$-NMR technique [14 16]. The number of possible implantation sites is one for ${ }^{8} \mathrm{Li}$ in $\mathrm{Zn}$ and two for ${ }^{8} \mathrm{~B}$ in $\mathrm{TiO}_{2}$. The relative populations are $90 \%$ for ${ }^{8} \mathrm{~B}$ implanted in the major site of $\mathrm{TiO}_{2}$ and $10 \%$ for that in the minor site. $\nu_{Q}$ and $\eta$ have been determined as $\nu_{Q}=+8.4 \pm 0.5 \mathrm{kHz}$ and $\eta=0$ for the implantation site of ${ }^{8} \mathrm{Li}$ in $\mathrm{Zn} \mathrm{[14]} \mathrm{and} \mathrm{as} \nu_{Q}=+144.5 \pm 0.6 \mathrm{kHz}$ and $\eta<0.03$ for the major implantation site of ${ }^{8} \mathrm{~B}$ in $\mathrm{TiO}_{2}$ [16]. Because of a small population for the minor site, it was difficult to detect a $\beta$-NQR signal for ${ }^{8} \mathrm{~B}$ in the minor site. $\nu_{Q}$ and $\eta$ at the minor site of ${ }^{8} \mathrm{~B}$ in $\mathrm{TiO}_{2}$ was evaluated as $\nu_{Q}=+1185 \pm 8 \mathrm{kHz}$ and $\eta=0.020 \pm 0.006$ from $\nu_{Q}$ at the minor site of ${ }^{12} \mathrm{~B}[15]$ and the ratio of the $Q$ moments of ${ }^{8} \mathrm{~B}$ and ${ }^{12} \mathrm{~B}[16]$. The directions of $q$ at an implantation site of ${ }^{8} \mathrm{Li}$ in $\mathrm{Zn}$ and at the major site of ${ }^{8} \mathrm{~B}$ in $\mathrm{TiO}_{2}$ [14, 16] were parallel to the $\mathrm{c}$ axis of the crystals, i.e., $\theta=0$, thus giving four frequencies split at regular intervals. For the minor site of ${ }^{8} \mathrm{~B}$ in $\mathrm{TiO}_{2}$, the direction of $q$ was inclined at $106^{\circ}$ relative to the $\langle 100\rangle$ axis on the (001) plane and the direction of $V_{\mathrm{YY}}$ was parallel to the c axis of the crystal [15], i.e., $\theta=\phi=90^{\circ}$, as the c axis was parallel to $B_{0}$. Frequencies for ${ }^{8} \mathrm{~B}$ at the major and the minor sites are shown as a function of $B_{0}$ in Fig. 3. Frequencies for the major site were isolated from those of the minor site only for the experimental condition of $B_{0}=230$ $\mathrm{mT}$. Under this condition, only the nuclear spin of ${ }^{8} \mathrm{~B}$ implanted in the major site can be manipulated. The $\beta$-ray angular distribution from the unmanipulated ${ }^{8} \mathrm{~B}$ in the minor site was stable. Because the alignment correlation term was derived from the dependence of the $\beta$-ray angular distribution on the degree of the alignment, the effect of ${ }^{8} \mathrm{~B}$ in the minor site was canceled.

The spin-aligning procedure for $\operatorname{spin} J=2$ was newly developed as part of our study. Figure 4 shows the schematic aligning procedure using ${ }^{8} \mathrm{Li}$ as an example. Immediately after the pulsed beam was stopped, the nuclear spin was manipulated by applying two kinds of 


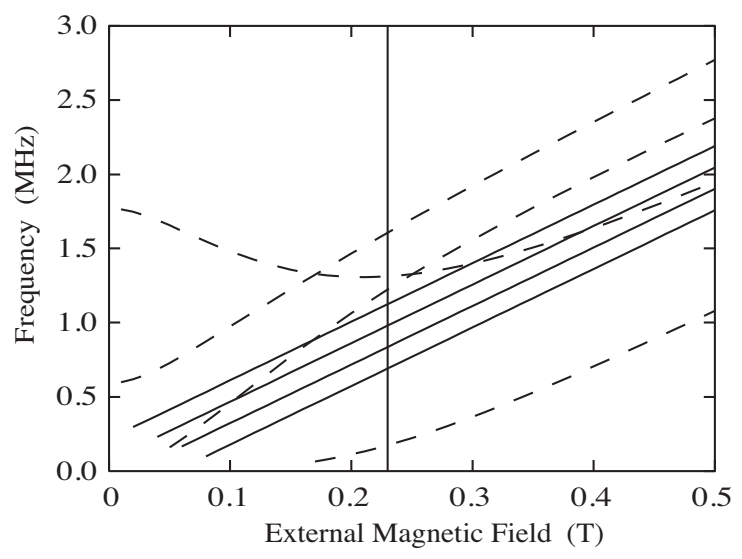

FIG. 3. External magnetic field dependence of the resonance frequencies of ${ }^{8} \mathrm{~B}$ in $\mathrm{TiO}_{2}$. The solid and dashed lines denote the frequencies of major and minor sites, respectively.

$\beta$-NMR technique, the adiabatic fast passage (AFP) and the depolarization methods. The populations between the two neighboring magnetic substates were interchanged by the AFP method and equalized by the depolarization method. To convert a positive polarization to a positive alignment $\mathcal{A}^{+}$, the populations in $m=+2$ and +1 , as well as in $m=-1$ and 0 were first of all equalized using the depolarization method. Following this, the positive alignment was produced by sequentially applying the AFP method four times, by which the populations between $m=+1$ and $0, m=-1$ and $0, m=-2$ and -1 , as well as $m=-1$ and 0 were interchanged. A negative alignment was produced immediately after the beam was stopped in the next beam-count cycle following a similar procedure applied to the magnetic substates as shown in the $\mathcal{A}^{-}$part of Fig. 4, For ${ }^{8} \mathrm{~B}$, an opposite sign of alignment was produced using the same procedure as for ${ }^{8} \mathrm{Li}$, because the polarization initially obtained for ${ }^{8} \mathrm{~B}$ was negative while the other parameters, i.e., the direction of the holding magnetic field and the field gradient, were similar. The alignment was converted back to a polarization to check the spin manipulation and to measure the relaxation time of the alignment. Subsequently, in the same beam-count cycle, the polarization was converted to an alignment with the opposite sign, as shown in Fig. 5. This method of data acquisition using the present timing program removed the systematic uncertainty due to beam fluctuation as described in Sec. IVB, 


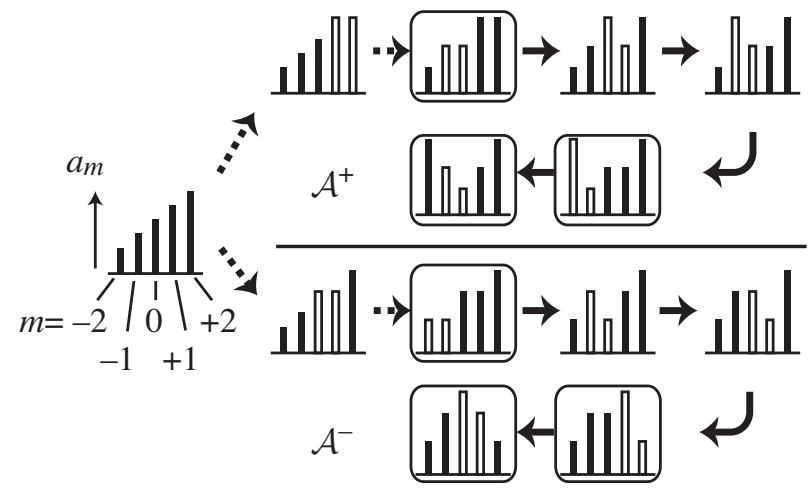

FIG. 4. Spin-aligning procedure for ${ }^{8} \mathrm{Li}$. The change in the populations, $a_{m}$, of the magnetic substate are shown. The spin manipulations with the AFP and depolarization methods of the NMR technique are denoted by the solid and dashed arrows, respectively. The two open bars in each orientation show the manipulated populations. The upper and lower parts show the production procedure for the positive and negative alignments, respectively. The polarizations of the three orientation patterns framed by the separate squares were measured to determine the alignment. The timing program for the measurement is shown in Fig. 5 ,

\section{Beta-ray energy spectra}

The $\beta$ rays were detected by two sets of plastic scintillation counter telescopes placed above $\left(\theta_{I \beta}=0^{\circ}\right)$ and below $\left(180^{\circ}\right)$ the crystal as shown in Fig. 1, Each telescope consisted of two thin $\Delta \mathrm{E}(\mathrm{A}$ and $\mathrm{B}$ ) detectors of $12 \mathrm{~mm} \phi \times 0.5 \mathrm{~mm}$ and $55 \mathrm{~mm} \phi \times 1 \mathrm{~mm}$, one $\beta$-ray energy (E) detector of $160 \mathrm{~mm} \phi \times 120 \mathrm{~mm}$, and one cone-shaped veto $(\overline{\mathrm{C}})$ detector. The $\overline{\mathrm{C}}$ detector was used to reject the $\beta$ rays scattered at the magnet. A typical counting rate of $\beta$ rays from $\beta$ emitters stopped in the catcher was 4 kcps $(1.5 \mathrm{kcps})$.

The energy spectra of $\beta$-rays emitted from purely aligned ${ }^{8} \mathrm{Li}$ and ${ }^{8} \mathrm{~B}$ are shown in Fig. 6 , The gain in the analog signal was stabilized using the standard light pulse from a lightemitting-diode (LED) pulser whose the circuit was maintained at a constant temperature.

The energy deposit in the E detector for a monoenergetic $\beta$ ray was obtained by a Monte Carlo simulation with the EGS4 code [17]. The detector telescopes, the catcher of the reaction products ${ }^{8} \mathrm{Li}$ and ${ }^{8} \mathrm{~B}$, the catcher holder, and the vacuum chamber near the $\beta$ ray window were arranged in the simulation. The distribution of the reaction products on the catcher was given using the reaction kinematics. The response function was obtained by convoluting the deposit function with a detector resolution as shown in Fig. 7. The 
(1)

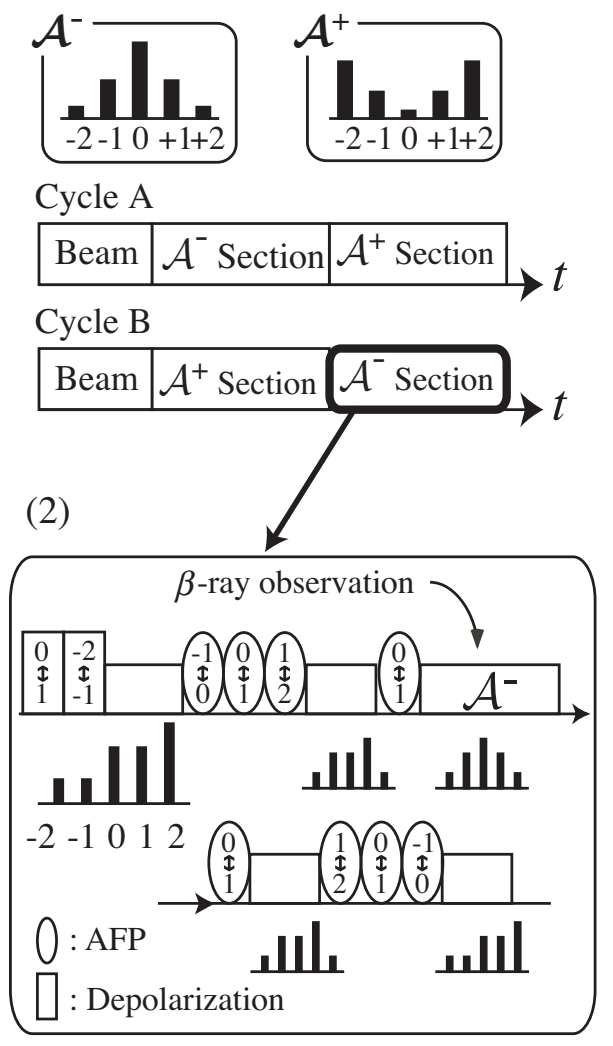

FIG. 5. Timing program for the spin-aligning process. In each cycle, the positive and negative alignments were produced as shown in (1). The timing program for the spin manipulation and the $\beta$-ray-angular-distribution measurement are shown in (2). The ellipses and squares with two numbers show the spin manipulation with the AFP and depolarization methods, respectively, used for the relevant transition between the magnetic substate nominally shown by the two numbers. The $\beta$-ray angular distribution was observed at the half-height squares. The alignment was converted back to the polarized form to check the spin manipulation and to measure alignment relaxation.

resolution of the Gaussian function was determined by the $\chi^{2}$ fitting of the $\beta$-ray energy spectra of ${ }^{8} \mathrm{Li}$ and ${ }^{8} \mathrm{~B}$ with $\sigma=\sigma_{0} \sqrt{E_{d e p}}$, where $\sigma_{0}=0.10 \pm 0.02(\mathrm{MeV})^{1 / 2}$. Here, $E_{\text {dep }}$ is the energy deposit in the $\mathrm{E}$ detector, which was observed, whereas the alignment correlation term needed to be extracted as a function of the $\beta$-ray energy just as it was emitted from the nucleus. The peak position of the energy deposit for monoenergetic $\beta$ rays was scaled to the incident energy of the $\beta$-ray. The $\beta$-ray energy spectrum for the $\chi^{2}$ fitting was obtained by convoluting the $\beta$-ray continuous energy spectrum with the response function of the monoenergetic $\beta$ ray. 


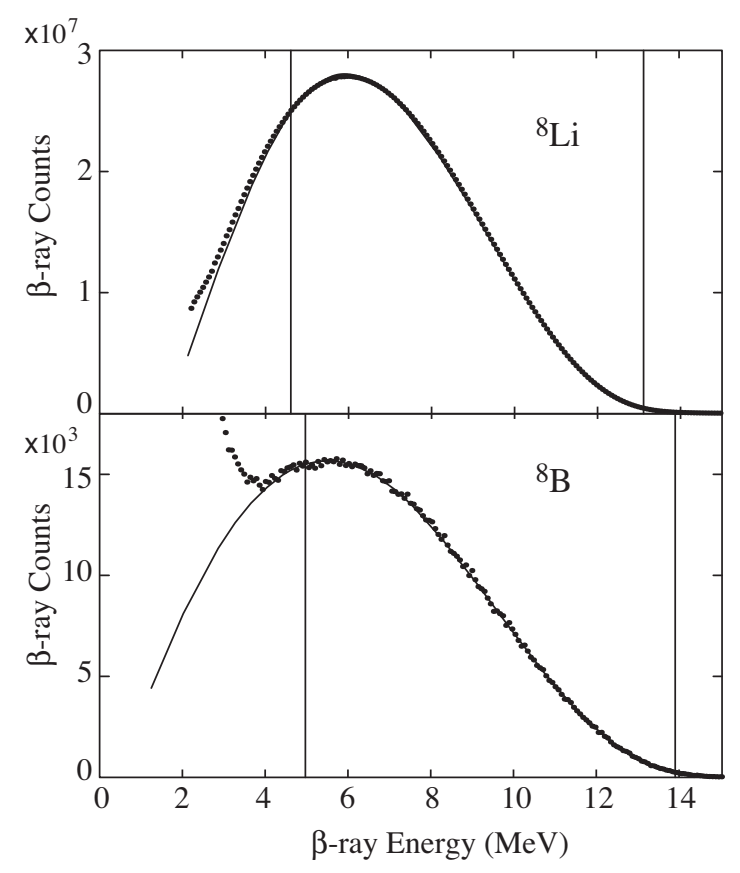

FIG. 6. Typical $\beta$-ray energy spectra for ${ }^{8} \mathrm{Li}$ (upper) and ${ }^{8} \mathrm{~B}$ (lower). The dots are the experimental data and the solid curves are the best-fit lines. The energy region lying between the two vertical lines in each energy spectrum shows the region used for the line fitting. The background $\beta$ rays in the low energy region of the ${ }^{8} \mathrm{~B}$ spectrum were from ${ }^{15} \mathrm{O}$.

The $\beta$-ray energy was scaled by determining the end-point energies of several $\beta$-emitters, which were ${ }^{8} \mathrm{Li}$ itself, ${ }^{28} \mathrm{Al}\left(E_{0}=2.86 \mathrm{MeV}\right),{ }^{20} \mathrm{~F}(5.39 \mathrm{MeV})$, and ${ }^{12} \mathrm{~B}(13.37 \mathrm{MeV})$ for the ${ }^{8} \mathrm{Li}$ experiment, and ${ }^{8} \mathrm{~B}$ itself, ${ }^{15} \mathrm{O}(1.73 \mathrm{MeV}),{ }^{20} \mathrm{~F}(5.39 \mathrm{MeV})$, and ${ }^{12} \mathrm{~N}(16.32 \mathrm{MeV})$ for the ${ }^{8} \mathrm{~B}$ experiment.

\section{ANALYSIS}

First, the determination of the degree of polarization and alignment, and then that of the alignment correlation terms are described. The evaluation of corrections and systematic uncertainties follows.

\section{A. Degree of polarization and alignment}

The polarization was determined from the $\beta$-ray asymmetry where $\beta$ rays from 5 to $13 \mathrm{MeV}$ were used. The $\beta$-ray angular distribution from the polarized nuclei is given by 


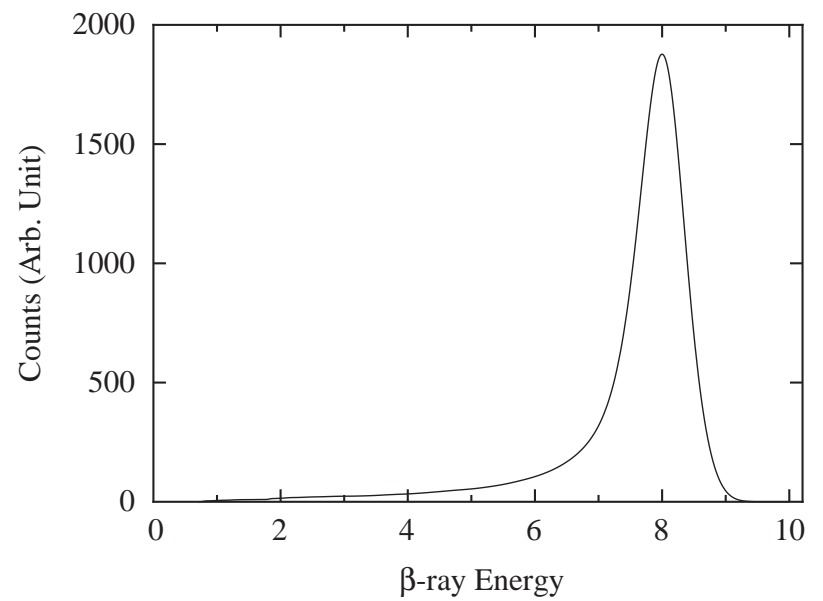

FIG. 7. Response function of the $\mathrm{E}$ detector for $\beta^{-}$ray with $8 \mathrm{MeV}$. The horizontal axis has been rescaled from the simulated energy deposit in the $\mathrm{E}$ detector to the $\beta$-ray energy just after the emission from the nucleus.

$W\left(\theta_{I \beta}\right) \propto B_{0}(E)+B_{1}(E) \mathcal{P} \cos \theta_{I \beta} \propto 1+A_{s} \mathcal{P} \cos \theta_{I \beta}$, where $\mathcal{P}$ is the degree of the polarization. The asymmetry parameter, $A_{s}=B_{1}(E) / B_{0}(E)$, has an energy independent-main term and an energy-dependent higher order term. For the determination of the degree of the polarization and alignment, $A_{s}$ was approximated as $-1 / 3$ for ${ }^{8} \mathrm{Li}$ and $+1 / 3$ for ${ }^{8} \mathrm{~B}$. The effect on the alignment correlation term by the higher order term of $A_{s}$ was corrected as described in Sec. IVC2, The counting ratio of the top and bottom telescopes, i.e. $\theta_{I \beta}=0^{\circ}$ and $180^{\circ}$, was caused by the $\beta$-ray asymmetry from the polarization $\mathcal{P}$ as well as the possible geometrical asymmetry $g$ resulting from the geometrical misalignment between two telescopes. This ratio is expressed as $R_{\beta}=W\left(0^{\circ}\right) / W\left(180^{\circ}\right)=g\left(1+A_{s} \mathcal{P}\right) /\left(1-A_{s} \mathcal{P}\right)$. To determine $g$, the polarization was inverted by applying a series of 10 AFPs. The measured counting ratios for the initial polarization $R_{\mathcal{P}_{+}}$, the inverted polarization $R_{\mathcal{P}_{-}}$, and the twice inverted polarization $R_{\mathcal{P}++}$ are given by

$$
\begin{aligned}
R_{\mathcal{P}+} & =g\left(1+A_{s} \mathcal{P}_{0}\right) /\left(1-A_{s} \mathcal{P}_{0}\right) \\
R_{\mathcal{P}-} & =g\left(1+\alpha A_{s} \mathcal{P}_{0}\right) /\left(1-\alpha A_{s} \mathcal{P}_{0}\right) \\
R_{\mathcal{P}++} & =g\left(1+\alpha^{2} A_{s} \mathcal{P}_{0}\right) /\left(1-\alpha^{2} A_{s} \mathcal{P}_{0}\right)
\end{aligned}
$$

From these equations, the initial polarization, $\mathcal{P}_{0}, g$ and the polarization inversion efficiency $\alpha$ were deduced, as shown in Table I. The inversion efficiency $\eta$ for the populations between the two magnetic substates by one AFP were determined from the relationship between $\alpha$ 
and $\eta, \alpha \approx 4-5 \eta$. The relaxation time of the polarization $T_{1}$ was determined from the time spectrum of polarization. These parameters are given in Table I.

The extraction of the degree of alignment from the negative alignment section shown in Fig. 4 was performed as follows. The $\beta$-ray asymmetry of the three orientations shown in Fig. 4 was observed during the aligning process. The polarization was determined from the measured asymmetry and $g$, as shown in Fig. 8. The population of the magnetic substate at the first orientation is given by $\left[a_{-2}, a_{-1}, a_{0}, a_{+1}, a_{+2}\right]=\left[r\left(1-\epsilon_{1}\right), r\left(1+\epsilon_{1}\right), s\left(1-\epsilon_{2}\right), s(1+\right.$ $\left.\left.\epsilon_{2}\right), t\right]$. The parameters $r, s$, and $t$ satisfy the relation $2 r+2 s+t=1 . \epsilon_{1}$ and $\epsilon_{2}$ are the parameters describing incompleteness in the depolarization method for the two different frequencies. These two parameters yielded a small residual polarization at the pure alignment section. The polarization of the first orientation is given by $\mathcal{P}_{1 \mathrm{st}}=\frac{1}{2}\left\{r\left(\epsilon_{1}-3\right)+s\left(\epsilon_{2}+1\right)+2 t\right\}$. The population after the spin manipulation using the AFP method, for example, between $m=+2$ and $m=+1$ is given as a matrix:

$$
\left(\begin{array}{ccccc}
1-\eta & \eta & 0 & 0 & 0 \\
\eta & 1-\eta & 0 & 0 & 0 \\
0 & 0 & 0 & 0 & 0 \\
0 & 0 & 0 & 0 & 0 \\
0 & 0 & 0 & 0 & 0
\end{array}\right)\left(\begin{array}{c}
a_{+2} \\
a_{+1} \\
a_{0} \\
a_{-1} \\
a_{-2}
\end{array}\right)
$$

where $\eta \approx 1$. The spin manipulation shown in Fig. 44(2) can be described as the product of the matrices. Therefore, the population at each orientation is given by $r, s, t, \eta, \epsilon_{1}$, and $\epsilon_{2}$. The polarization at the second orientation is given by $\mathcal{P}_{2 \text { nd }} \approx \frac{1}{2}\left\{4 r \epsilon_{1}+s\left(\epsilon_{2}-1\right)+\right.$ $\left.t+(1-\eta)\left(-6 r\left(\epsilon_{1}+1\right)-s\left(\epsilon_{2}-5\right)+t\right)\right\}$ under the approximation, up to the first order, that $(1-\eta) \ll 1$. The pure alignment is produced at the third orientation. The residual polarization is given by $\mathcal{P}_{3 \mathrm{rd}} \approx \frac{1}{2}\left\{4 r \epsilon_{1}+2 s \epsilon_{2}+(1-\eta)\left(-6 r\left(\epsilon_{1}+1\right)-3 s\left(\epsilon_{2}-1\right)+3 t\right)\right\}$. $\eta$ was determined from the measurement of $R_{\mathcal{P}^{+}}, R_{\mathcal{P}^{-}}$, and $R_{\mathcal{P}^{++}}$. Therefore, the number of free parameters is three by assuming $\epsilon_{1}=\epsilon_{2}$ and giving the relation $2 r+2 s+t=1$. All the population parameters were determined from the polarization change of the three orientations.

The alignment in the third orientation can be calculated from the population parameters using $\mathcal{A} \approx \frac{1}{2}\left\{-2+8 r+2 s+(1-\eta)\left(-10 r\left(\epsilon_{1}+1\right)-s\left(\epsilon_{2}-5\right)+5 t\right\}\right.$. This equation gives the alignment prior to the alignment section. In order to consider the alignment relaxation in the crystal, the alignments prior to and after the alignment section were determined 


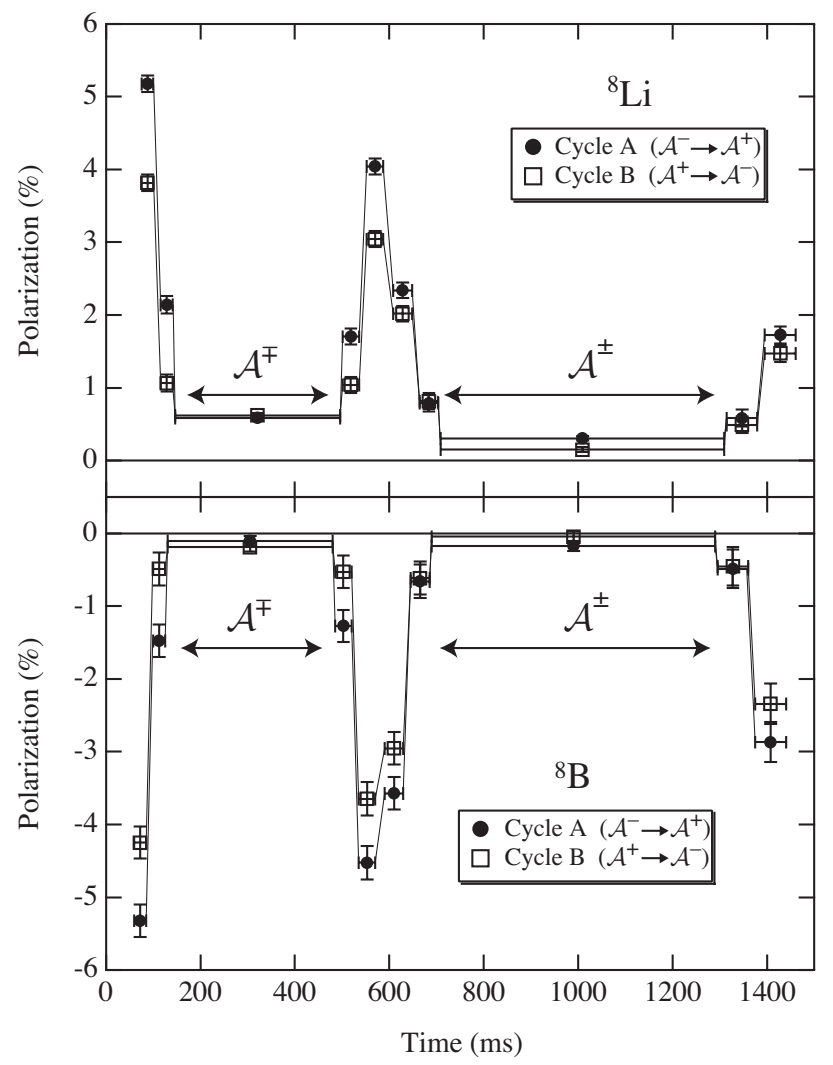

FIG. 8. Polarization change in the timing program for the spin-aligning process. The filled circles and the open squares are for the cycles A and B in Fig. 5, respectively. The beam was chopped and was stopped for the cycle at the time 0 .

from the polarization change before and after the alignment section, respectively. Then the effective alignment and the relaxation time of the alignment were deduced. Using a different assumption that $\epsilon_{1}=10 \epsilon_{2}$ or $\epsilon_{1}=0.1 \epsilon_{2}$, the systematic uncertainty was estimated. The change in the alignment was less than the statistical uncertainty. The results for the spin manipulation are summarized in Table 【.

\section{B. Alignment correlation term}

The alignment correlation term was obtained from the ratio of counts, $R(E)=N\left(E, d \mathcal{P}^{+}, \mathcal{A}^{+}\right) / N(E, d \mathcal{P}$ at the positive and negative alignment sections. $\mathcal{A}$ and $d \mathcal{P}$ are the alignment and the residual polarization at the alignment section, respectively. The signs given by the superscript in $\mathcal{A}^{ \pm}$ and $d \mathcal{P}^{ \pm}$are the alignment signs. The counts are proportional to the $\beta$-ray angular distribution as expressed by $\left.N(E, d \mathcal{P}, \mathcal{A}) \propto B_{0}(E)\left[1 \pm\left(B_{1}(E) / B_{0}(E)\right) d \mathcal{P}+\left(B_{2}(E) / B_{0}(E)\right) \mathcal{A}\right)\right]$, 
TABLE I. Results of the spin manipulation. $\mathcal{P}_{0}$ is the initial polarization. $\mathcal{A}_{1 / 2}^{\mp}$ is the alignment, where subscripts 1 and 2 indicate the first and second halves of the timing program, respectively, and the superscript is the sign of the alignment. $\Delta \mathcal{A}_{1+2}$ is the sum of the absolute value of the alignments. $T_{1}$ and $T_{\mathcal{A}}$ are the relaxation times of the polarization and the alignment, respectively. $\alpha$ and $\eta$ are the efficiencies of the polarization inversion and the population inversion between the two neighboring magnetic substates. $\epsilon$ is a parameter of the incompleteness of the depolarization.

\begin{tabular}{ccc}
\hline \hline & ${ }^{8} \mathrm{Li}$ & ${ }^{8} \mathrm{~B}$ \\
\hline $\mathcal{P}_{0}(\%)$ & $7.18 \pm 0.10$ & $5.42 \pm 0.19$ \\
$\mathcal{A}_{1}^{+}(\%)$ & $+3.96 \pm 0.20$ & $+4.9 \pm 0.4$ \\
$\mathcal{A}_{1}^{-}(\%)$ & $-4.93 \pm 0.20$ & $-5.6 \pm 0.4$ \\
$\mathcal{A}_{2}^{+}(\%)$ & $+2.29 \pm 0.19$ & $+3.9 \pm 0.4$ \\
$\mathcal{A}_{2}^{-}(\%)$ & $-1.91 \pm 0.19$ & $-3.2 \pm 0.4$ \\
$\Delta \mathcal{A}_{1+2}(\%)$ & $13.1 \pm 0.4$ & $17.7 \pm 0.8$ \\
$T_{1}(\mathrm{~s})$ & $13.0 \pm 1.6$ & $13 \pm 4$ \\
$T_{\mathcal{A}}(\mathrm{s})$ & $2.0 \pm 0.7$ & $2.2 \pm 1.2$ \\
$\alpha(\%)$ & $-85.5 \pm 0.3$ & $-94.8 \pm 0.9$ \\
$\eta(\%)$ & $97.09 \pm 0.07$ & $98.95 \pm 0.18$ \\
$\epsilon\left(10^{-3}\right)$ & $4.4 \pm 0.2$ & $-0.9 \pm 0.4$ \\
\hline \hline
\end{tabular}

where the upper and lower signs are for the top and bottom telescopes, respectively.

For the first half of the cycles $\mathrm{A}$ and $\mathrm{B}$ shown in Fig. 5 , the counting ratio $R_{1}(E)$ is given by

$$
R_{1}(E)=\frac{T^{+} N\left(E, d \mathcal{P}_{1}^{+}, \mathcal{A}_{1}^{+}\right)}{T^{-} N\left(E, d \mathcal{P}_{1}^{-}, \mathcal{A}_{1}^{-}\right)}
$$

where the values with subscript 1 , such as $\mathcal{A}_{1}^{+}$, are for the first half. $T^{-}$and $T^{+}$are the beam-current integral for the cycles $\mathrm{A}$ and $\mathrm{B}$, respectively. The alignment correlation term was derived using the well-approximated formula as

$$
R_{1}(E) \approx \frac{T^{+}}{T^{-}}\left\{1 \pm \frac{B_{1}(E)}{B_{0}(E)} d \mathcal{P}_{1}+\frac{B_{2}(E)}{B_{0}(E)} \Delta \mathcal{A}_{1}\right\}
$$

where the upper and lower signs are for the top and bottom telescopes, respectively. $d \mathcal{P}_{1}=$ $d \mathcal{P}_{1}^{+}-d \mathcal{P}_{1}^{-}$and $\Delta \mathcal{A}_{1}=\mathcal{A}_{1}^{+}-\mathcal{A}_{1}^{-}$. The ratio of $T^{+}$and $T^{-}$caused a spurious $\beta$-ray 
asymmetry in $R_{1}(E)$. The counting ratio $R_{2}(E)$ at the second half of the cycles $\mathrm{A}$ and $\mathrm{B}$ is given by

$$
\begin{aligned}
R_{2}(E) & =\frac{T^{-} N\left(E, d \mathcal{P}_{2}^{+}, \mathcal{A}_{2}^{+}\right)}{T^{+} N\left(E, d \mathcal{P}_{2}^{-}, \mathcal{A}_{2}^{-}\right)} \\
& =\frac{T^{-}}{T^{+}}\left\{1 \pm \frac{B_{1}(E)}{B_{0}(E)} d \mathcal{P}_{2}+\frac{B_{2}(E)}{B_{0}(E)} \Delta \mathcal{A}_{2}\right\}
\end{aligned}
$$

where the values with subscript 2 , such as $\mathcal{A}_{2}^{+}$, are for the second half. In the double ratio $R_{1}(E) R_{2}(E), T^{+}$and $T^{-}$are canceled as

$$
\begin{aligned}
R_{1}(E) R_{2}(E)= & \frac{N\left(E, d \mathcal{P}_{1}^{+}, \mathcal{A}_{1}^{+}\right)}{N\left(E, d \mathcal{P}_{2}^{-}, \mathcal{A}_{2}^{-}\right)} \frac{N\left(E, d \mathcal{P}_{2}^{+}, \mathcal{A}_{2}^{+}\right)}{N\left(E, d \mathcal{P}_{2}^{-}, \mathcal{A}_{2}^{-}\right)} \\
\approx & 1 \pm \frac{B_{1}(E)}{B_{0}(E)} d \mathcal{P}_{1+2} \\
& +\frac{B_{2}(E)}{B_{0}(E)} \Delta \mathcal{A}_{1+2},
\end{aligned}
$$

where $d \mathcal{P}_{1+2}=d \mathcal{P}_{1}+d \mathcal{P}_{2}$ and $\Delta \mathcal{A}_{1+2}=\Delta \mathcal{A}_{1}+\Delta \mathcal{A}_{2}$. The alignment correlation terms were extracted from the simple average of the double ratios $R_{1}(E) R_{2}(E)$ for the top and bottom telescopes so that the influence of the residual polarization was canceled.

\section{Corrections}

In the extraction procedure for the alignment correlation terms described above, the $\beta$-ray angular distribution for ${ }^{8} \mathrm{Li}$ is given by

$$
W(E) \propto p E\left(E_{0}-E\right)\left\{1 \mp \frac{1}{3} \mathcal{P}+\frac{B_{2}(E)}{B_{0}(E)} \mathcal{A}\right\},
$$

where the upper and lower signs are for telescopes with $\theta_{I \beta}=0^{\circ}$ and $180^{\circ}$, respectively, instead of the following:

$$
\begin{aligned}
W\left(E, \theta_{I \beta}\right) \propto & p E\left(E_{0}-E\right) \\
& \left\{1+\frac{B_{1}(E)}{B_{0}(E)} \frac{p}{E} \mathcal{P} P_{1}\left(\cos \left(\theta_{I \beta}\right)\right)\right. \\
& \left.+\frac{B_{2}(E)}{B_{0}(E)}\left(\frac{p}{E}\right)^{2} \mathcal{A} P_{2}\left(\cos \left(\theta_{I \beta}\right)\right)\right\} .
\end{aligned}
$$

The correction for the $P_{1}\left(\cos \left(\theta_{I \beta}\right)\right)$, and $P_{2}\left(\cos \left(\theta_{I \beta}\right)\right)$ is given in Sec. IVC1. The corrections for $(p / E),(p / E)^{2}$, and $B_{1}(E) / B_{0}(E)$ are given in Sec. IVC2. 


\section{Solid angle of $\beta$-ray telescope}

The polarization and alignment correlation terms in the $\beta$-ray angular distribution are proportional to the Legendre polynomials $P_{1}\left(\cos \left(\theta_{I \beta}\right)\right)$ and $P_{2}\left(\cos \left(\theta_{I \beta}\right)\right)$, i.e., the $\cos \theta_{I \beta}$ and $\frac{3}{2}\left(\cos ^{2} \theta_{I \beta}-1 / 3\right)$ terms, respectively. $R_{1}(E) R_{2}(E)$ in Eq. (12) includes $B_{2}(E) / B_{0}(E)$, so the $\frac{3}{2}\left(\cos ^{2} \theta_{I \beta}-1 / 3\right)$ contribution should be corrected. $\Delta \mathcal{A}_{1+2}$ was determined from the degree of polarization, so the $\cos \theta_{I \beta}$ contribution should be corrected. In order to take the finite solid angle of the detector into account, the detection efficiency as a function of $E$ and $\theta_{I \beta}$ was simulated using the EGS4 code. The correction for the solid angle was evaluated by convoluting the simulated efficiency, as shown in Fig. 9.

\section{Higher order term in the polarization and alignment correlation terms}

The $p / E$ term and the $B_{1}(E) / B_{0}(E)$ term in the polarization correlation term were assumed to be 1 and $\mp 1 / 3$, where the upper and lower signs are for ${ }^{8} \mathrm{Li}$ and ${ }^{8} \mathrm{~B}$, respectively, when the polarization was determined from the $\beta$-ray asymmetry. The correction for the polarization is independent of energy, because the polarization was determined from the total count from 5 to $13 \mathrm{MeV}$. The correction for the $p / E$ term was 0.9972 and 0.9973 for ${ }^{8} \mathrm{Li}$ and ${ }^{8} \mathrm{~B}$, respectively. The $B_{1}(E) / B_{0}(E)$ term is given in [3] as

$$
\begin{aligned}
\frac{B_{1}(E)}{B_{0}(E)}= & \mp \frac{1}{3}\left[1+\frac{E}{3 M_{n}}\left(\frac{1}{A} \pm \frac{b}{A c}-\frac{d_{\mathrm{I}}}{A c}\right)\right. \\
& -\frac{\sqrt{21}}{4}\left\{ \pm \frac{f}{A c} \frac{4 E+E_{0}+4 E^{2} / E_{0}}{3 M_{n}}\right. \\
& \left.\left.-\frac{j_{2}}{A^{2} c} \frac{8 E^{2}-5 E E_{0}}{2 M_{n}^{2}}\right\}\right] .
\end{aligned}
$$

To avoid the large systematic uncertainty from the $j_{2} / A^{2} c$ term, the correction factor was evaluated using the product of the correction factor at $\frac{5}{8} E_{0}$ and the ratio of the value at $\frac{5}{8} E_{0}$ to the averaged value from 5 to $13 \mathrm{MeV}$. The ratio was determined, from the observed energy dependence of the polarization correlation term, to be $0.983 \pm 0.007$ for ${ }^{8} \mathrm{Li}$ and $1.013 \pm 0.014$ for ${ }^{8} \mathrm{~B}$. The correction factor at $\frac{5}{8} E_{0}$ was self-consistently evaluated using iteration to be $0.98 \pm 0.03$ for ${ }^{8} \mathrm{Li}$ and $0.99 \pm 0.03$ for ${ }^{8} \mathrm{~B}$ from the matrix elements $b / A c$, $d_{\mathrm{I}} / A c, f / A c$, and $j_{2} / A^{2} c$, which in the present study were determined from the alignment correlation terms and the $\beta$ - $\alpha$ angular correlation terms. The uncertainty of this correction 
included the uncertainty of the matrix elements and a 100\% uncertainty of the higher order contribution from $f / A c$, thus implying a severe evaluation. Accordingly, the correction factor for $B_{1}(E) / B_{0}(E)$ was $0.96 \pm 0.03$ for ${ }^{8} \mathrm{Li}$ and $1.00 \pm 0.03$ for ${ }^{8} \mathrm{~B}$.

The $(p / E)^{2}$ term in the alignment correlation term is assumed to be 1 for the first-order analysis. The evaluated correction factor for the $(p / E)^{2}$ term is shown in Fig. 9.

\section{Detector response}

The observed alignment correlation term includes the contribution from the neighboring energy region to some extent because of the finite detector resolution and the low-energy tail component of the detector response, as shown in Fig. 17. The correction factor was evaluated self-consistently using the known detector response and the alignment correlation term, as shown in Fig. 9. Here, the alignment correlation term was approximated by a quadratic curve, $c_{1} E+c_{2} E^{2}$, with two parameters $c_{1}$ and $c_{2}$. The correction factor for ${ }^{8} \mathrm{~B}$ from 6 to 12 $\mathrm{MeV}$ was close to 1.0 because the alignment correlation term was almost constant and the influence of the different energy was small.

\section{Background}

The main backgrounds for ${ }^{8} \mathrm{Li}$ and ${ }^{8} \mathrm{~B}$ below $4 \mathrm{MeV}$ were ${ }^{17} \mathrm{~F}\left(T_{1 / 2}=64.5 \mathrm{~s}, Q_{E C}=\right.$ $2.76 \mathrm{MeV})$ and ${ }^{15} \mathrm{O}\left(T_{1 / 2}=122 \mathrm{~s}, Q_{E C}=2.75 \mathrm{MeV}\right)$, respectively. The correction for the background is also shown in Fig. 9. The systematic uncertainty in the alignment correlation term was estimated by assuming $20 \%$ ambiguity in the background fraction.

\section{Systematic uncertainties}

In this subsection, the systematic uncertainties of the alignment correlation term are described. They are also summarized in Table II.

\section{Polarization relaxation and rank-three spin orientation}

The polarization and alignment were relaxed as a function of time. While the alignment relaxation during the alignment correlation term measurement was taken into account in the 


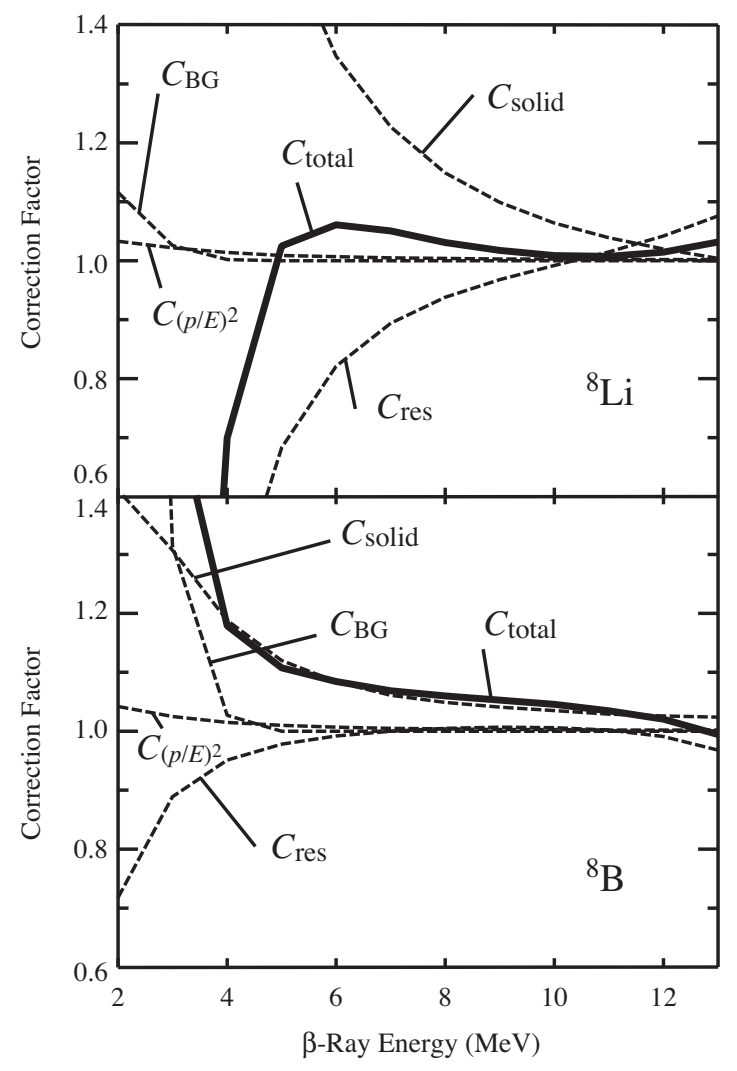

FIG. 9. Energy dependent correction factors and total correction for ${ }^{8} \mathrm{Li}$ (upper) and ${ }^{8} \mathrm{~B}$ (lower). The correction factors for the solid angle, $C_{\text {solid }}$, the detector response, $C_{\text {res }}$, the background, $C_{\mathrm{BG}}$, and the $(p / E)^{2}$ term in the $\beta$-ray angular distribution, $C_{(p / E)^{2}}$, are shown. The total correction factor, $C_{\text {total }}$ also includes the energy independent correction factors for the $(p / E)$ and $B_{1} / B_{0}$ terms in the $\beta$-ray angular distribution.

procedure of the alignment extraction, the relaxation during conversion from the polarization to alignment was evaluated as a systematic uncertainty.

The $\beta$-ray asymmetry was caused by the odd rank of the spin orientation. The degree of the rank-three spin orientation was determined from the population parameters similar to the case of the degree of alignment, as described in Sec. IVA. The polarization was evaluated by taking the degree of the rank-three orientation into account. The effect on the polarization was considered as a systematic uncertainty. 


\section{Uncertainty in the correction factor resulting from self-consistent evaluation}

The correction factor for the detector response was self-consistently evaluated using the results of the alignment correlation term. The energy dependence of the alignment correlation term was estimated as a quadratic curve without a constant term. The statistical uncertainty of the quadratic curve was propagated to the systematic uncertainty.

The correction factor for $B_{1}(E) / B_{0}(E)$ was evaluated using the matrix elements, such as $d_{\mathrm{I}} / A c$. The systematic uncertainty for this correction factor has been discussed in Sec. IV C.

\section{Relative positions of the beam, recoil catcher, and telescope}

The implanted recoil nuclei distributed widely on the catcher except for the part in the shadow part of the collimator. The relative positions of the beam spot and the catcher were able to change the distribution of the reaction products on the catcher. The beam spot was tuned using a fluorescent target with accuracy $0.5 \mathrm{~mm}$ and $1 \mathrm{~mm}$ in the horizontal and vertical directions, respectively. The relative position of the catcher and the $\Delta \mathrm{E}$ detector had the potential to change the solid angle of the telescope and $\beta$-ray efficiency. The uncertainty of this relative position was $2 \mathrm{~mm}$. The correction factors were evaluated by using the detector response simulated for different conditions using the EGS4 code. The change in the alignment correlation terms because of these two uncertainties was considered to be the systematic uncertainty.

\section{Detector response function}

The reliability of the low-energy tail in the simulated response function of a monoenergetic $\beta$-ray was evaluated. The low-energy tail was mainly caused by the energy loss straggling in the material between the positions of the $\beta$-ray emitter and E detector. The largest uncertainty was due to the catcher thickness. The relative uncertainty of the thickness was $10 \%$ for all the crystals.

The reliability of the simulated low-energy tail has been studied experimentally [18]. ${ }^{12} \mathrm{~B}$ and ${ }^{12} \mathrm{~N}$ were produced as emitters. The -ray energy was selected via a dipole magnet. The shape and amount of low-energy tail were confirmed to within $20 \%$ statistical uncertainty. 
The catcher thickness uncertainty of $10 \%$ and the simulation reliability of $20 \%$ for the low-energy tail were simulated simultaneously by varying the crystal thickness by $30 \%$ in the EGS4 simulation. The correction factors were evaluated by simulating the detector response at a crystal thickness varied by $30 \%$. The systematic uncertainty in the alignment correlation terms was evaluated using these correction factors.

The detector resolution was determined from that reproducing the most complete experimental $\beta$-ray spectra of ${ }^{8} \mathrm{Li}$ and ${ }^{8} \mathrm{~B}$. The uncertainty of the counter resolution was $20 \%$. The correction factors were evaluated using counter resolutions both the $20 \%$ larger and $20 \%$ smaller than the most probable resolution. The systematic uncertainty in the alignment correlation terms was evaluated using these correction factors.

\section{Energy scaling, gain fluctuation, and pileup}

The systematic uncertainty due to the energy-scale uncertainty $\delta E$ was evaluated using

$\frac{d}{d E}\left(B_{2}(E) / B_{0}(E)\right) \delta E . B_{2}(E) / B_{0}(E)$ was given by the polynomial for $E$ and $E^{2}$, where the coefficients were determined by the $\chi^{2}$ fit analysis.

The gain fluctuation was typically within $40 \mathrm{keV}$. The systematic uncertainty due to the gain fluctuation of the E detector was evaluated using the same procedure as that for the energy-scale uncertainty.

For a pileup event caused by two $\beta$ rays, the obtained alignment correlation term is determined on the basis of the contributions of the two $\beta$ rays at their respective energies. This effect was evaluated as a systematic uncertainty by integrating its contribution over the energy of two $\beta$ rays.

\section{RESULTS AND DISCUSSION}

After the obtained alignment correlation terms are shown, the results given by Eq. (2) are compared with the CVC prediction. And then the weighted mean value of end-point energy over final-state distribution is described, which is used when the matrix elements are determined from the alignment correlation terms and the $\beta$ - $\alpha$ correlation terms. Finally, the extraction of the weak magnetism and the second-forbidden term is described and these terms are compared with the CVC prediction. 
TABLE II. Systematic uncertainties of the alignment correlation term at $9 \mathrm{MeV}$.

\begin{tabular}{ccc}
\hline \hline & ${ }^{8} \mathrm{Li}$ & ${ }^{8} \mathrm{~B}$ \\
& $\times 10^{-2}$ & $\times 10^{-2}$ \\
\hline Polarization relaxation & 0.002 & 0.003 \\
Third-order orientation & 0.015 & 0.026 \\
Uncertainty of $B_{2} / B_{0}$ in & & 0.007 \\
detector-response correction & 0.010 & 0.133 \\
Uncertainty of matrix & & 0.027 \\
elements in $B_{1} / B_{0}$ correction & 0.107 & 0.039 \\
Position of beam spot and catcher & & 0.034 \\
Position of $\Delta$ E detector and catcher & 0.011 & 0.017 \\
Low-energy tail of detector response & 0.015 & 0.001 \\
Detector resolution & 0.050 & $<0.001$ \\
Energy scaling & 0.021 & 0.001 \\
Gain fluctuation & 0.053 & 0.148 \\
Pileup & 0.028 & 0.001 \\
\hline \hline
\end{tabular}

\section{A. Alignment correlation terms and $\beta$ - $\alpha$ correlation terms}

The alignment correlation terms that were obtained are shown in Fig. 10, The statistical uncertainty of the alignment $\Delta \mathcal{A}_{1+2}$ in Eq. (12) could shift all data points of the alignment correlation term in the same direction. The statistical uncertainty of $\Delta \mathcal{A}_{1+2}$ is not included in each data point of Fig. 10 in order to retain a statistical fluctuation among the different points; however, the statistical uncertainties of the final results, such as $a_{\mathrm{WM}}^{\beta}$, include the statistical uncertainty of $\Delta \mathcal{A}_{1+2}$.

The $\beta$ - $\alpha$ correlation terms $-\frac{2}{3} p_{ \pm}(E)$ [11] are also shown as crosses. The weak magnetism term, $-\left(3 M_{n} / 4 E\right)\left(\delta_{\text {align. }}^{-}+\delta_{\beta \alpha}^{-}\right)=b / A c$, was derived combining the two types of correlation term shown in Fig. 11. Figure 11] also reflects a reanalysis using the same energy bin as the $\beta$ - $\alpha$ correlation terms [11]. 


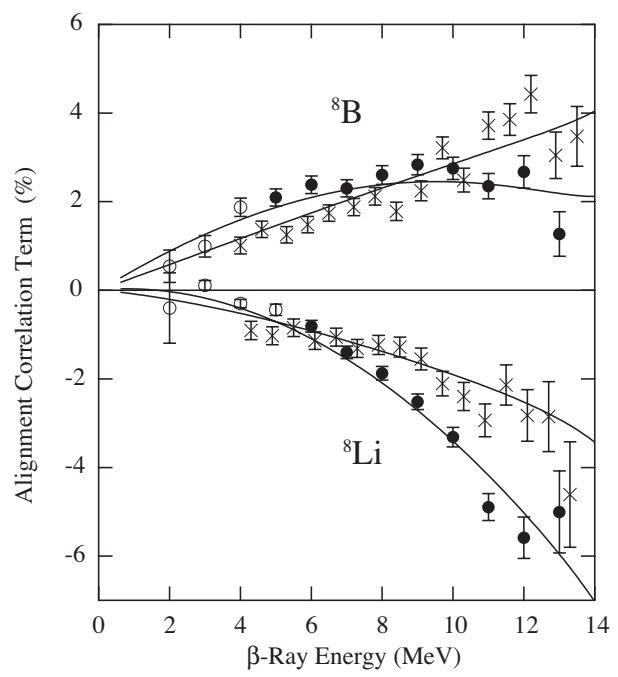

FIG. 10. Alignment correlation terms and $\beta$ - $\alpha$ correlation terms. The circles are the alignment correlation terms and the crosses are the $\beta$ - $\alpha$ angular correlation terms. The open circles are not used for the derivation of the matrix elements. The lines are the best-fit curves.

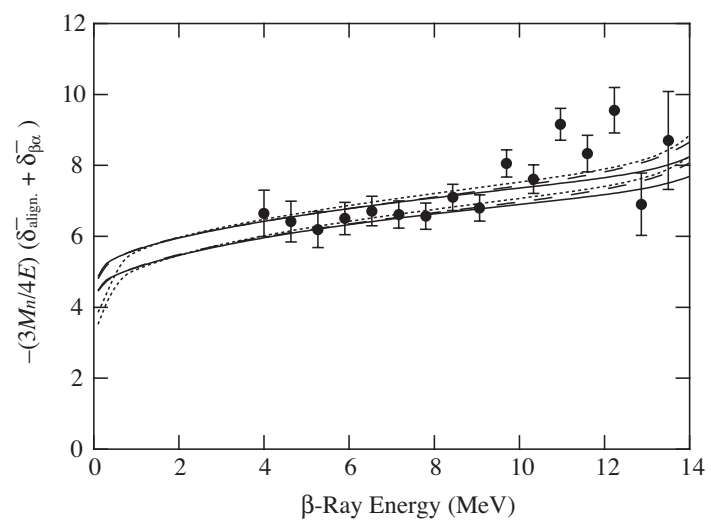

FIG. 11. Weak magnetism term derived from the $\beta$-ray correlation terms. The CVC predictions are shown by the $1 \sigma$ error band. The solid, dashed and dotted bands are the present, De Braeckeleer's [7], and Winter's [19, 20] predictions, respectively. Winter's prediction was re-evaluated using the mirror-averaged end-point energy.

\section{B. CVC prediction}

The experimental $b / A c$ results shown in Fig. 11]indicates a slight $E$ dependence. The CVC prediction of an energy dependent $b / A c$ has in previous studies been indicated and described by introducing the dependence into the matrix elements, $b\left(E_{x}\right)$ and $c\left(E_{x}\right)$, of the final-state energy $E_{x}$ in ${ }^{8} \mathrm{Be}$ [7, 21-23]. The final-state energy distributes widely because several states 
with spin and parity of $2^{+}$are mixed because of the wide decay width. This final-state distribution can be formulated using the $R$-matrix theory with four final states [24, 25]. We re-evaluated the CVC prediction in Ref. [12] by using the analog- $\gamma$-decay measurement by De Braeckeleer et al. [7], and the recent measurement of the $\beta$-delayed- $\alpha$ energy spectra from ${ }^{8} \mathrm{Li}$ and ${ }^{8} \mathrm{~B}$ by Bhattacharya et al. [25]. The procedure for this re-evaluation was same as for the previous work [7] except for the number of final states; three final states were used in it, while four final states were used in the present evaluation, similar to that for the Gamow-Teller matrix element $c\left(E_{x}\right)$ in Ref. [25]. The procedure is summarized below.

The $E_{x}$ dependence of $c\left(E_{x}\right)$ gives the final-state distribution for the $\beta$ decay, i.e., the delayed $\alpha$ energy spectrum. The mirror-averaged $c\left(E_{x}\right)$ was determined from the delayed $\alpha$ energy spectra of ${ }^{8} \mathrm{Li}$ and ${ }^{8} \mathrm{~B}$ based on the $R$-matrix formalism by Bhattacharya et al. [25].

$b\left(E_{x}\right)$ is given by the isovector M1 transition strength of the analog- $\gamma$ decay, based on the strong CVC. The isobaric analog state in ${ }^{8} \mathrm{Be}$ was produced using the ${ }^{4} \mathrm{He}(\alpha, \gamma)$ reaction and the de-excited $\gamma$ ray was measured [7]. The $E_{x}$ dependence of $b\left(E_{x}\right)$ gives the finalstate distribution in the analog- $\gamma$ decay, which has been measured through the $\gamma$-ray energy spectra shown in Fig. 4 of Ref. [7]. The matrix elements, $\mathcal{M}_{1}^{\gamma}$ and $R_{\gamma}$ in $b\left(E_{x}\right)$, gives the $E_{x}$ dependence of $b\left(E_{x}\right)$ and were determined using three final states in Ref. [7]. The $\mathcal{M}_{1}^{\gamma}$ is the weak magnetism matrix element for the transition to the first excited state, and $R_{\gamma}$ is the ratio, $\mathcal{M}_{16}^{\gamma} / \mathcal{M}_{1}^{\gamma}$, where $\mathcal{M}_{16}^{\gamma}$ represents the transition to an isospin doublet at $16 \mathrm{MeV}$. These matrix elements were re-determined for the four final states so as to reproduce the $\gamma$-ray energy spectra, which were $\mathcal{M}_{1}^{\gamma}=8.71 \pm 0.28$ and $R_{\gamma}=1.5 \pm 1.4$.

The $E$ dependent $b / A c$ is given in [7] by the weighted average as

$$
\frac{b}{A c} \rightarrow \frac{\int b\left(E_{x}\right) c\left(E_{x}\right)\left(E_{\max }-E_{x}-E\right) d E_{x}}{A \int c^{2}\left(E_{x}\right)\left(E_{\max }-E_{x}-E\right) d E_{x}}
$$

Here, $c\left(E_{x}\right)$ and $E_{\max }$ averaged between the mirror pair were used, because $b / A c$ was derived from the $\beta$-ray angular correlations of both ${ }^{8} \mathrm{Li}$ and ${ }^{8} \mathrm{~B}$. The $\mathrm{CVC}$ prediction of $b / A c$ determined from Eq. (16) is shown in Fig. 11,

The CVC prediction determined by De Braeckeleer et al. [7] and Winter et al. [19, 20] are also shown in Fig. 11. The previous predictions have a problem in regards to final-state treatment. The delayed- $\alpha$ spectra have been reproduced very well using four final states [24, 25]. De Braeckeleer et al., however, used three states for both $b\left(E_{x}\right)$ and $c\left(E_{x}\right)$, and Winter et al. used three states only for $b\left(E_{x}\right)$. The present CVC prediction was slightly 
smaller than the previous predictions at a higher energy region.

The transition to the first excited state of ${ }^{8} \mathrm{Be}$ was predominant for the analog- $\gamma$ transition [7]. Therefore, for a comparison between the $b / A c$ extracted from the $\beta$ decay and its CVC prediction, the weak magnetism for the first excited state, $a_{\mathrm{WM}}$, was used. This was given by the matrix elements of the transition to the first excited state, $a_{\mathrm{WM}}^{\beta}=\mathcal{M}_{1}^{\beta} / A \mathcal{M}_{\mathrm{GT} 1}^{\beta}$ for $\beta$ decay and $a_{\mathrm{WM}}^{\mathrm{CVC}}=\mathcal{M}_{1}^{\gamma} / A \mathcal{M}_{\mathrm{GT} 1}^{\beta}$ for the CVC prediction, where $\mathcal{M}_{1}^{\beta}$ and $\mathcal{M}_{\mathrm{GT} 1}^{\beta}$ are the weak magnetism, $b$, and Gamow-Teller, $c$, matrix elements for the weak transition to the first excited state, respectively. The expression for $b / A c$ using $\mathcal{M}_{1}$ and $\mathcal{M}_{\mathrm{GT} 1}$ was given in Ref. [7]. The CVC prediction was determined to be $a_{\mathrm{WMC}}^{\mathrm{CVC}}=7.3 \pm 0.2$ based on $\mathcal{M}_{1}^{\gamma}=8.71 \pm 0.28$ and the mirror-averaged $\mathcal{M}_{\mathrm{GT} 1}^{\beta}=0.1496 \pm 0.0005$ [25]. The CVC prediction of $f$ was determined by the isovector $\mathrm{M} 1 / \mathrm{E} 2$ ratio $\delta_{1}=0.01 \pm 0.03$ [7] as $a_{\mathrm{WE} 2}^{\mathrm{CVC}}=\sqrt{10 / 3} \delta_{1} a_{\mathrm{WM}}^{\mathrm{CVC}}=0.1 \pm 0.4$. The values are summarized in Tables III and IV.

TABLE III. Decay widths and matrix elements for the $\gamma$ decay from the isobaric analog state in ${ }^{8} \mathrm{Be} . \Gamma_{M 1}^{T=1}$ is the decay width for the isovector component of the M1 transition from the isobaric analog state $(T=1) . \delta_{1}$ is the isovector M1/E2 ratio from the isobaric analog state. Definition of $\mathcal{M}_{1}^{\gamma}$ and $R_{1}^{\gamma}$ is described in the text. Average value $\mathcal{M}_{\mathrm{GT} 1}^{\beta}$ of the Gamow-Teller matrix elements of ${ }^{8} \mathrm{Li}$ and ${ }^{8} \mathrm{~B}$ is also shown.

\begin{tabular}{lclc}
\hline \hline Analog $\gamma$ decay & Value & Matrix Element & Value \\
\hline$\Gamma_{M 1}^{T=1}[7]$ & $2.80 \pm 0.18 \mathrm{eV}$ & $\mathcal{M}_{1}^{\gamma}[\underline{7}]^{\mathrm{a}}$ & $8.7 \pm 0.3$ \\
$\Gamma_{M 1}^{T=1}[23]^{\mathrm{b}}$ & $3.6 \pm 0.3 \mathrm{eV}$ & $\mathcal{M}_{1}^{\gamma}[23]^{\mathrm{c}}$ & $9.9 \pm 0.6$ \\
$\Gamma_{M 1}^{T=1}[22]^{\mathrm{b}}$ & $4.1 \pm 0.6 \mathrm{eV}$ & $\mathcal{M}_{1}^{\gamma}[22]^{\mathrm{c}}$ & $10.5 \pm 0.9$ \\
$\delta_{1}[7]$ & $0.01 \pm 0.03$ & $R_{1}^{\gamma}[7]^{\mathrm{a}}$ & $1.5 \pm 1.4$ \\
$\delta_{1}[23]^{\mathrm{b}}$ & $0.14 \pm 0.03$ & $\mathcal{M}_{\mathrm{GT} 1}^{\beta}[25]$ & 0.1496 \\
& & & \pm 0.0005 \\
\hline \hline
\end{tabular}

\footnotetext{
${ }^{\text {a }}$ Reanalyzed in the present work using the four final states in the $R$-matrix formalism.

b Reanalyzed in Ref. [7].

${ }^{c}$ Calculated from $\mathcal{M}_{1}^{\gamma}$ of Refs. [7] and $\Gamma_{M 1}^{T=1}$ of Ref. [7, 23] or Refs. [7, 22] using the relation $\mathcal{M}_{1}^{\gamma} \propto \sqrt{\Gamma_{M 1}^{T=1}}$.
} 


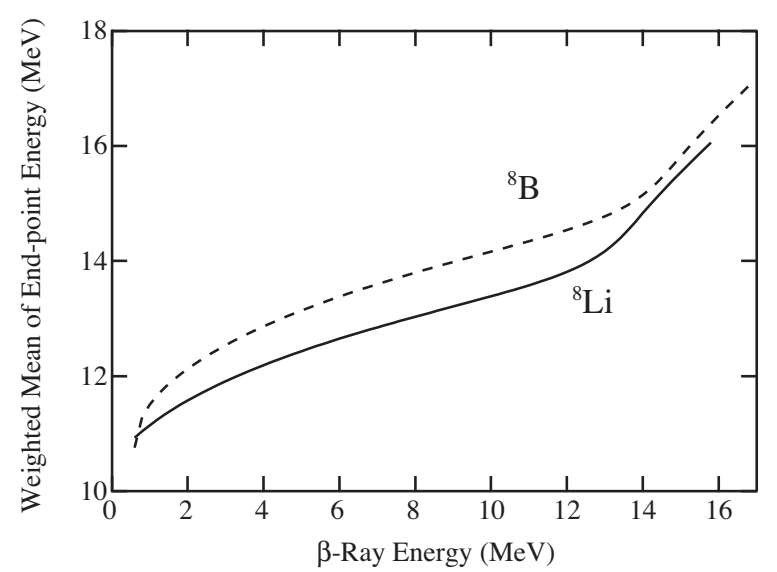

FIG. 12. Weighted mean value of the end-point energy over the broad-final-state distribution as a function of $\beta$-ray energy.

\section{End-point energy}

The end-point energy of the $\beta$ ray is not a constant because of the broad final state. The alignment correlation terms and the $\beta$ - $\alpha$ correlation terms given in Eq. (1) were measured as a function of $\beta$-ray energy without measurement of the end-point energy. Therefore, the end-point energy was averaged over the final-state-energy distribution. The weight is the product of $p E\left(E_{0}\left(E_{x}\right)-E\right)^{2}$ and the final-state distribution $c^{2}\left(E_{x}\right)$ of the $\beta$ decay. When a certain $\beta$-ray energy is chosen, the weighted mean value of the end-point energy is given by

$$
\begin{aligned}
\bar{E}_{0}(E) & =\frac{\int p E\left(E_{0}\left(E_{x}\right)-E\right)^{2} c^{2}\left(E_{x}\right) E_{0}\left(E_{x}\right) d E_{x}}{\int p E\left(E_{0}\left(E_{x}\right)-E\right)^{2} c^{2}\left(E_{x}\right) d E_{x}} \\
& =\frac{\int\left(E_{0}\left(E_{x}\right)-E\right)^{2} c^{2}\left(E_{x}\right) E_{0}\left(E_{x}\right) d E_{x}}{\int\left(E_{0}\left(E_{x}\right)-E\right)^{2} c^{2}\left(E_{x}\right) d E_{x}},
\end{aligned}
$$

where $E_{0}\left(E_{x}\right)=E_{\max }-E_{x}$ and the integral range is from 0 to $\left(E_{\max }-E\right)$. Figure 12 shows $\bar{E}_{0}(E)$ calculated using $c\left(E_{x}\right)$ determined in Ref. [25]. This $\bar{E}_{0}(E)$ was used in the analysis to determine the matrix elements.

\section{Weak magnetism and second-forbidden terms from the weak vector current}

The mirror difference $\delta^{-}$consists of $b / A c$ and a small contribution of $j_{2} / A^{2} c$ due to the mirror asymmetry of $E_{0}$. To avoid the influence of this mirror asymmetry, the $\chi^{2}$ fit analysis was performed simultaneously on the four correlation terms, i.e., both alignment correlation terms and $\beta-\alpha$ angular correlation terms of ${ }^{8} \mathrm{Li}$ and ${ }^{8} \mathrm{~B}$. The $E_{x}$ dependent $b / A c$ in the 
$\beta$-ray angular correlation terms was given by the same formula as the CVC prediction of $b / A c . \quad a_{\mathrm{WM}}^{\beta}=\mathcal{M}_{1}^{\beta} / A \mathcal{M}_{\mathrm{GT} 1}^{\beta}$ was used as a free parameter for the $\chi^{2}$ fit analysis, where $R_{\beta}$ was assumed to be the same as $R_{\gamma}$. The $E$ dependences of $a_{\mathrm{WE} 2}^{\beta}, d_{\mathrm{I}} / A c, j_{2} / A^{2} c$, and $j_{3} / A^{2} c$ were not clearly seen in the $\beta$-ray correlation terms because of the relatively large statistical uncertainties. These terms were assumed to be constant and were chosen as free parameters for the $\chi^{2}$ fit analysis. The obtained terms were considered as the value averaged over the analyzed energy region. The best-fit curves are shown in Fig. 10 and the results are summarized in Table IV. The weak magnetism and the second-forbidden terms were $a_{\mathrm{WM}}^{\beta}=7.54 \pm 0.12$ (stat.) \pm 0.15 (syst.) and $a_{\mathrm{WE} 2}^{\beta}=1.0 \pm 0.2$ (stat.) \pm 0.2 (syst), respectively. The systematic uncertainty because of the $E$ dependence of $a_{\mathrm{WE} 2}^{\beta}$ was estimated to be 0.05 for $a_{\mathrm{WE} 2}^{\beta}$ by assuming that the $E_{x}$ dependence of $f\left(E_{x}\right)$ was the same as $b\left(E_{x}\right)$. The other systematic uncertainties in the alignment correlation terms and the $\beta$ - $\alpha$ correlation terms were independently propagated to those in $a_{\mathrm{WM}}^{\beta}$ and $a_{\mathrm{WE} 2}^{\beta}$ by performing the $\chi^{2}$ fit analysis for the data applied to the different correction factors. $a_{\mathrm{WM}}^{\beta}$ was consistent with the CVC prediction from De Braeckeleer's data, i.e., $a_{\mathrm{WM}}^{\beta} / a_{\mathrm{WM}}^{\mathrm{CVC}}=1.03 \pm 0.04$. However, the present $a_{\mathrm{WE} 2}^{\beta}$ is inconsistent with the De Braeckeleer's data, $a_{\mathrm{WE} 2}^{\mathrm{CVC}}=0.1 \pm 0.4$. The deviation of $a_{\mathrm{WE} 2}$ was $1.8 \sigma$ as $a_{\mathrm{WE} 2}^{\beta}-a_{\mathrm{WE} 2}^{\mathrm{CVC}}=0.9 \pm 0.5$.

TABLE IV. Ratio of matrix elements contributing to the $\beta$-ray angular correlations. The CVC predictions are also shown. $a_{\mathrm{WM}}^{\mathrm{CVC}}=\mathcal{M}_{1}^{\gamma} / A \mathcal{M}_{\mathrm{GT} 1}^{\beta}$ and $a_{\mathrm{WE} 2}^{\mathrm{CVC}}=\sqrt{10 / 3} \delta_{1} a_{\mathrm{WM}}^{\mathrm{CVC}}$.

\begin{tabular}{lclc}
\hline \hline Matrix Element & Value & Matrix Element & Value \\
\hline$a_{\mathrm{WM}}^{\beta}$ & $7.5 \pm 0.2$ & $a_{\mathrm{WM}}^{\mathrm{CVC}}[7]$ & $7.3 \pm 0.2$ \\
$a_{\mathrm{WE} 2}^{\beta}$ & $1.0 \pm 0.3$ & $a_{\mathrm{WM}}^{\mathrm{CVC}}[23]$ & $8.3 \pm 0.5$ \\
$d_{\mathrm{I}} / A c$ & $5.5 \pm 1.7$ & $a_{\mathrm{WM}}^{\mathrm{CVC}}[22]$ & $8.8 \pm 0.7$ \\
$j_{2} / A^{2} c$ & $-490 \pm 70$ & $a_{\mathrm{WE} 2}^{\mathrm{CVC}}[\underline{7}$ & $0.1 \pm 0.4$ \\
$j_{3} / A^{2} c$ & $-980 \pm 280$ & $a_{\mathrm{WE} 2}^{\mathrm{CVC}}[23]$ & $2.1 \pm 0.5$ \\
\hline \hline
\end{tabular}

We compared these results with the other analog- $\gamma$-decay measurements by Bowles and Garvey [23] and Paul et al. [22]. The CVC prediction was $a_{\mathrm{WM}}^{\mathrm{CVC}}=8.3 \pm 0.5$ and $a_{\mathrm{WE} 2}^{\mathrm{CVC}}=$ $2.1 \pm 0.5$ for Bowles' data, and $a_{\mathrm{WM}}^{\mathrm{CVC}}=8.8 \pm 0.7$ for Paul's data. These predictions were inconsistent with the De Braeckeleer's data and also with the present $\beta$-decay results; that is, both $a_{\mathrm{WM}}^{\mathrm{CVC}}$ were larger than $a_{\mathrm{WM}}^{\beta}$, and the deviation of $a_{\mathrm{WE} 2}$ was $1.8 \sigma$, as $-1.1 \pm 0.6$. It 
was pointed out by De Braeckeleer et al. [7] that there were problems in these measurements in regards to the absolute cross section, the photon angular distribution and the neutron background. The difference between the two $a_{\mathrm{WE} 2}^{\mathrm{CVC}}$ was due to deviation of $\delta_{1}$, i.e., $0.01 \pm$ 0.03 [7] compared to $0.14 \pm 0.03$ [7, 23]. This deviation was determined via the relatively difficult measurement of the photon angular distribution. The inconsistency might be due to an underestimated background for the photon angular distribution. Although the CVC prediction by De Braeckeleer et al. was adopted in the present work, De Braeckeleer's data need to be confirmed with more accurate measurements.

\section{SUMMARY}

The nuclear-spin-aligned nuclei ${ }^{8} \mathrm{Li}$ and ${ }^{8} \mathrm{~B}$ were produced from spin-polarized nuclei using the $\beta$-NMR technique to test the strong CVC at a zero momentum transfer limit. The strong CVC could be tested for the second-forbidden transition for the first time. The alignment correlation terms for the $\beta$-ray angular distribution were determined using both positively and negatively aligned nuclei. The weak magnetism and the second-forbidden terms originating from the weak vector current were determined by combining the present alignment correlation terms and the previously known $\beta$ - $\alpha$ angular correlation terms. The CVC predictions of the weak magnetism and the second-forbidden terms were re-evaluated using the most precise data set of the analog- $\gamma$ decay in ${ }^{8}$ Be. Although the weak magnetism term was consistent with the CVC prediction obtained from the isovector-M1-transition strength, the second-forbidden term was inconsistent with that from the isovector-E2-transition strength. For more reliable tests for the second-forbidden transition, the CVC predictions need to be confirmed by more accurate measurements especially with regard to the isovector M1/E2 ratio $\delta_{1}$.

\section{ACKNOWLEDGMENTS}

This work was supported by KAKENHI (21740189).

[1] See e.g., I.S. Towner and J.C. Hardy, in Symmetries and Fundamental Interactions in Nuclei, 
edited by W.C. Haxton and E.M. Henley (World Scientific, Singapore, 1995), nucl-th/9504015.

[2] J.C. Hardy and I.S. Towner, Phys. Rev. C 79, 055502 (2009).

[3] B.R. Holstein, Rev. Mod. Phys. 46, 789 (1974).

[4] L. Grenacs, Ann. Rev. Nucl. Part. Sci. 35, 455 (1985).

[5] Y. Lee, L. Mo and C. Wu, Phys. Rev. Lett. 10, 253 (1963).

[6] K. Minamisono, K. Matsuta, T. Minamisono, T. Yamaguchi, T. Sumikama, T. Nagatomo, M. Ogura, T. Iwakoshi, M. Fukuda, M. Mihara, K. Koshigiri, and M. Morita, Phys. Rev. C 65, 015501 (2002).

[7] L. De Braeckeleer, E. G. Adelberger, J. H. Gundlach, M. Kaplan, D. Markoff, A. M. Nathan, W. Schieff, K. A. Snover, D. W. Storm, K. B. Swartz, D. Wright, and B. A. Brown, Phys. Rev. C 51, 2778 (1995).

[8] K. Minamisono, et al., Phys. Rev. C (to be submitted).

[9] Y.G. Abov et al., Nucl. Phys. 34, 505 (1962); H. Eichner et al., Z. Naturforsh. 21a, 908 (1966).

[10] R.E. Tribble and G.T. Garvey, Phys. Rev. C 12, 967 (1975).

[11] R.D. McKeown, G.T. Garvey, and C.A. Gagliardi, Phys. Rev. C 22, 738 (1980).

[12] T. Sumikama, K. Matsuta, T. Nagatomo, M. Ogura, T. Iwakoshi, Y. Nakashima, H. Fujiwara, M. Fukuda, M. Mihara, K. Minamisono, T. Yamaguchi, and T. Minamisono, Phys. Lett. B 664, 235 (2008).

[13] A. Abragam, Principles of Nuclear Magnetism (Oxford, New York, 1983).

[14] T. Ohtsubo, Y. Nakayama, I. Minami, M. Tanigaki, S. Fukuda, A. Kitagawa, M. Fukuda, K. Matsuta, Y. Nojiri, H. Akai, and T. Minamisono, Hyperfine Interact. 80, 1051 (1993).

[15] T. Sumikama, M. Ogura, Y. Nakashima, T. Iwakoshi, M. Mihara, M. Fukuda, K. Matsuta, T. Minamisono, and H. Akai, Hyperfine Interact. 158, 413 (2004).

[16] T. Sumikama, T. Nagatomo, M. Ogura, T. Iwakoshi, Y. Nakashima, H. Fujiwara, K. Matsuta, T. Minamisono, M. Fukuda, and M. Mihara, Phys. Rev. C 74, 024327 (2006).

[17] W.R. Nelson and H. Hirayama and D.W.O. Rogers, Stanford University Report, SLAC-265; D.W.O. Rogers, Nucl. Instr. and Meth. A227, 535 (1984).

[18] K. Tanaka, M. Fukuda, M. Mihara, K. Kidera, A. Takemura, H. Kenzaki, K. Matsuta, and T. Minamisono, OULNS Ann. Rep., p. 38 (1998).

[19] W. T. Winter, S. J. Freedman, K. E. Rehm, I. Ahmad, J. P. Greene, A. Heinz, D. Henderson, R. V. F. Janssens, C. L. Jiang, E. F. Moore, G. Mukherjee, R. C. Pardo, T. Pennington, 
G. Savard, J. P. Schiffer, D. Seweryniak, G. Zinkann, and M. Paul, Phys. Rev. Lett. 91, 252501 (2003).

[20] W. T. Winter, S. J. Freedman, K. E. Rehm, and J. P. Schiffer, Phys. Rev. C 73, 025503 (2006), nucl-ex/0406019.

[21] A. M. Nathan, G. T. Garvey, P. Paul, and E. K. Warburton, Phys. Rev. Lett. 35, 1137 (1975).

[22] P. Paul, M. Suffert, and P. Gorodetzky, Phys. Lett. B 71, 71 (1975).

[23] T. J. Bowles and G. T. Garvey, Phys. Rev. C 18, 1447 (1978).

[24] E. K. Warburton, Phys. Rev. C 33, 303 (1986).

[25] M. Bhattacharya, E. G. Adelberger, and H. E. Swanson, Phys. Rev. C 73, 055802 (2006). 